Modelling the Shallow Water Equations in Curvilinear Coordinates with Physical Application

S. Wingenter

January 27, 2005 
This document was prepared as an account of work sponsored by an agency of the United States Government. Neither the United States Government nor the University of California nor any of their employees, makes any warranty, express or implied, or assumes any legal liability or responsibility for the accuracy, completeness, or usefulness of any information, apparatus, product, or process disclosed, or represents that its use would not infringe privately owned rights. Reference herein to any specific commercial product, process, or service by trade name, trademark, manufacturer, or otherwise, does not necessarily constitute or imply its endorsement, recommendation, or favoring by the United States Government or the University of California. The views and opinions of authors expressed herein do not necessarily state or reflect those of the United States Government or the University of California, and shall not be used for advertising or product endorsement purposes.

This work was performed under the auspices of the U.S. Department of Energy by University of California, Lawrence Livermore National Laboratory under Contract W-7405-Eng-48. 


\title{
MODELLING THE SHALLOW WATER EQUATIONS IN CURVILINEAR COORDINATES WITH PHYSICAL APPLICATION
}

\author{
A Thesis \\ Presented to the \\ Faculty of \\ San Diego State University
}

\author{
In Partial Fulfillment \\ of the Requirements for the Degree \\ Master of Science \\ in \\ Computational Science
}

by

Suzanne Wingenter

November 12004 


\section{THE UNDERSIGNED FACULTY COMMITTEE APPROVES \\ THE THESIS OF SUZANNE WINGENTER:}

\begin{tabular}{c}
\hline Jose Castillo, Chair \\
Computational Science Research Center \\
\hline Ricardo Carretero \\
Department of Mathematics and Statistics \\
\hline Victor M. Ponce \\
Department of Civil Engineering \\
\hline Carlos Torres \\
Universidad Autonoma De Baja California
\end{tabular}

Date

Universidad Autonoma De Baja California

SAN DIEGO STATE UNIVERSITY

November 12004 


\section{ACKNOWLEDGEMENTS}

It is amazing the lengths to which scientific achievement has grown through computational experimentation, but there still must be strict adherence to physical behavior in the real world. No matter how much further we are to go down this road of replacing expensive physical experimentation with cheaper computational simulation, no study should be complete without some validation of results. But as fields of study narrow, and with the advent of multi-disciplinary degrees, there is not enough time to do everything without collaborative efforts.

Thanks to Dr. Jose Castillo for the encouragement and patience. Thanks to Dr. Carlos Torres for the endless hours of oceanography explanation. Thanks to Dr. Victor M. Ponce for planting the seed that would grow into this project, and providing a wonderful starting point. In addition, thanks to Dr. Ricardo Careterro for always supplying a fresh perspective. Finally, I wish to thank Dr. Petri Fast of Lawrence Livermore National Laboratory for all his generous advice.

Work on the simulations done with Overture in Chapter 6 were performed at Lawrence Livermore National Laboratory during the summer of 2004. I wish to thank the Lab for the wonderful opportunity. This document has been reviewed and released under UCRL number: $* * * * * * * * * * *$. 


\section{TABLE OF CONTENTS}

PAGE

ACKNOWLEDGEMENTS $\ldots \ldots \ldots \ldots \ldots \ldots \ldots \ldots \ldots \ldots \ldots \ldots \ldots \ldots \ldots \ldots \ldots \ldots \ldots \ldots$

LIST OF TABLES $\ldots \ldots \ldots \ldots \ldots \ldots \ldots \ldots \ldots \ldots \ldots \ldots \ldots \ldots \ldots \ldots \ldots \ldots \ldots \ldots \ldots$

LIST OF FIGURES $\ldots \ldots \ldots \ldots \ldots \ldots \ldots \ldots \ldots \ldots \ldots \ldots \ldots \ldots \ldots \ldots \ldots \ldots \ldots \ldots \ldots \ldots$ CHAPTER

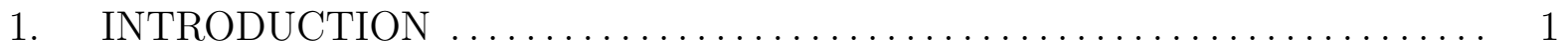

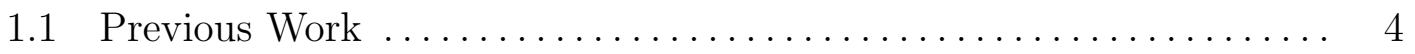

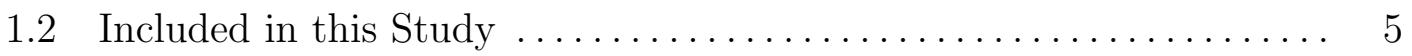

2. CARTESIAN EQUATIONS AND PROBLEM DEFINITION $\ldots \ldots \ldots \ldots \ldots \ldots$

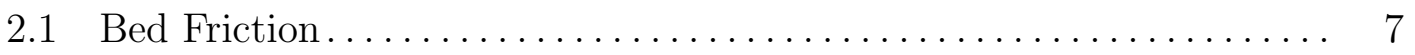

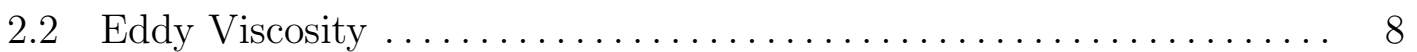

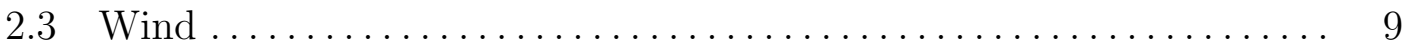

3. TRANSFORMING THE EQUATIONS $\ldots \ldots \ldots \ldots \ldots \ldots \ldots \ldots \ldots \ldots \ldots$

4. OVERVIEW OF THE NUMERICAL SCHEME $\ldots \ldots \ldots \ldots \ldots \ldots \ldots \ldots \ldots \ldots \ldots$

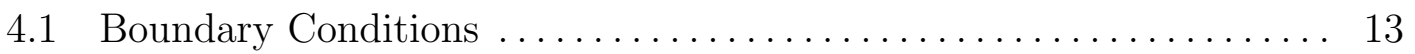

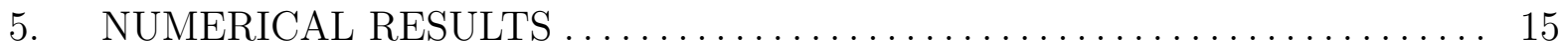

5.1 Propagation of a Surface Wave $\ldots \ldots \ldots \ldots \ldots \ldots \ldots \ldots \ldots \ldots \ldots \ldots$

5.1.1 Short Time Run Results . . . . . . . . . . . . . . . . . . 19

5.1 .2 Long Time Run Results ..................... 20

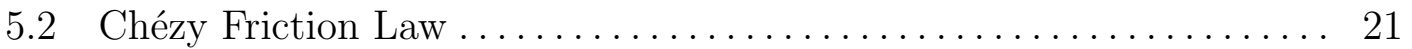


CHAPTER PAGE

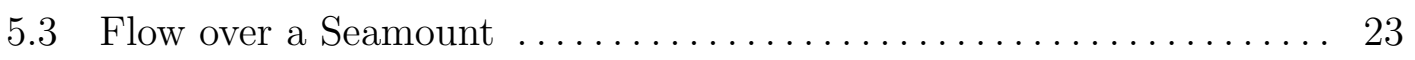

5.4 Generic River Geometry $\ldots \ldots \ldots \ldots \ldots \ldots \ldots \ldots \ldots \ldots \ldots \ldots \ldots \ldots \ldots \ldots \ldots \ldots \ldots$

5.5 Flow Past an Idealized Island ..................... 29

6. OVERTURE AND THE BAHIA DE TODOS SANTOS $\ldots \ldots \ldots \ldots \ldots \ldots$

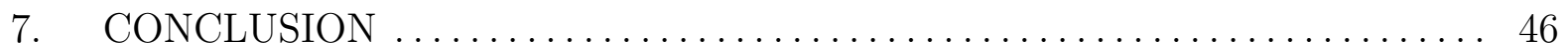

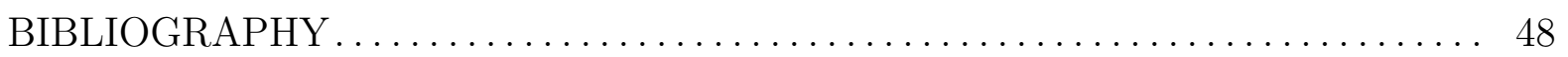

APPENDICES

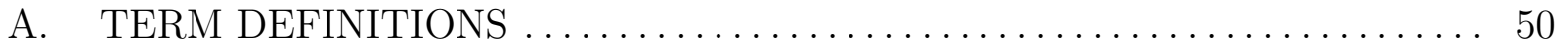

B. FINITE DIFFERENCE SCHEME - L OPERATORS $\ldots \ldots \ldots \ldots \ldots \ldots \ldots \ldots . . \ldots 2$

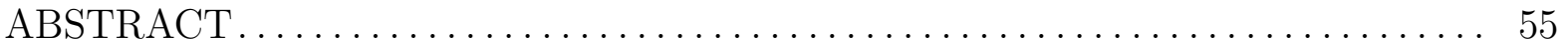




\section{LIST OF TABLES}

TABLE

PAGE

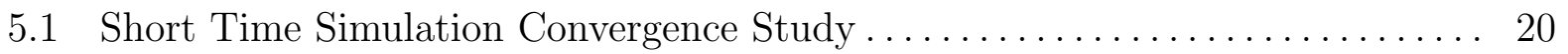

5.2 Long Time Simulation Convergence Study . . . . . . . . . . . . . . . . . . 20

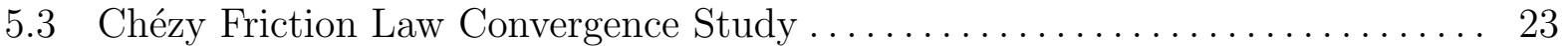




\section{LIST OF FIGURES}

FIGURE

PAGE

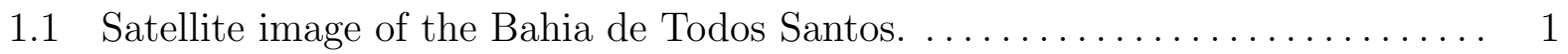

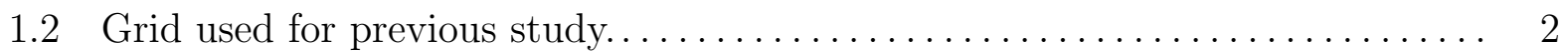

1.3 Transformation from Cartesian to curvilinear coordinates. . . . . . . . . . . 3

1.4 Overset grid of the Bahia de Todos Santos. $\ldots \ldots \ldots \ldots \ldots \ldots \ldots \ldots \ldots \ldots \ldots$

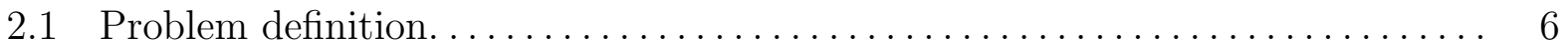

5.1 Curvilinear grid used for verification study, 100 x 50 meters. . . . . . . . . 18

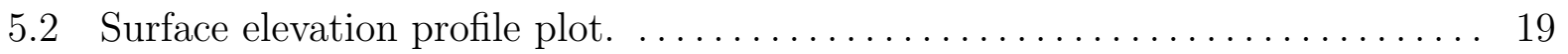

5.3 Surface elevation profile plot. $\ldots \ldots \ldots \ldots \ldots \ldots \ldots \ldots \ldots \ldots \ldots \ldots \ldots \ldots \ldots \ldots \ldots$

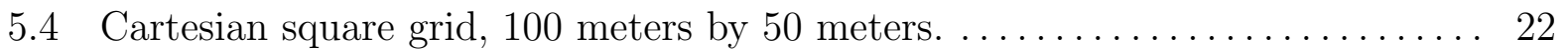

5.5 Bottom bathymetry and grid for flow past a seamount. . . . . . . . . . 24

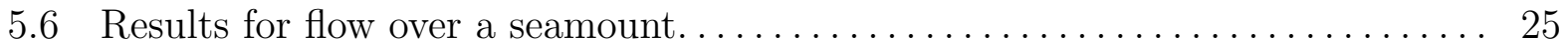

5.7 Generic river geometry grid. $\ldots \ldots \ldots \ldots \ldots \ldots \ldots \ldots \ldots \ldots \ldots \ldots \ldots \ldots \ldots \ldots \ldots$

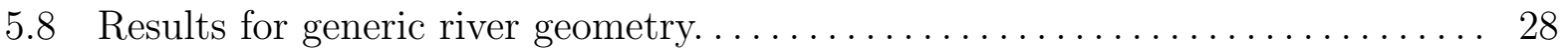

5.9 Annulus grid for flow past an idealized island $\ldots \ldots \ldots \ldots \ldots \ldots \ldots \ldots \ldots$

5.10 Plots for flow past an idealized island for eddy viscosity $20 \mathrm{~m}^{2} / \mathrm{s} \ldots \ldots \ldots 32$

5.11 Plots for flow past an idealized island for eddy viscosity $5 \mathrm{~m}^{2} / \mathrm{s} \ldots \ldots \ldots \ldots 33$

5.12 Plots for flow past an idealized island for eddy viscosity $1 \mathrm{~m}^{2} / \mathrm{s} . \ldots \ldots \ldots 34$

5.13 Plots for flow past an idealized island for eddy viscosity $0.5 \mathrm{~m}^{2} / \mathrm{s} \ldots \ldots \ldots 35$

6.1 Overset grid example, an annulus in a straight channel. $\ldots \ldots \ldots \ldots \ldots \ldots$ 
PAGE

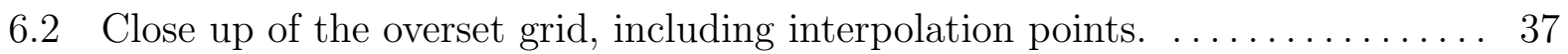

6.3 Overlapping grid of the Bahia de Todos Santos. $\ldots \ldots \ldots \ldots \ldots \ldots \ldots \ldots$

6.4 Previous study on Bahia de Todos Santos results. . . . . . . . . . . . . . . . 40

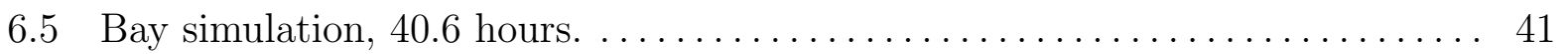

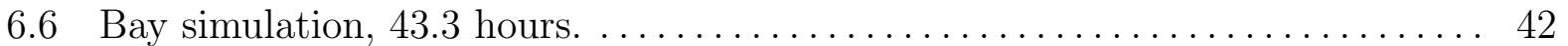

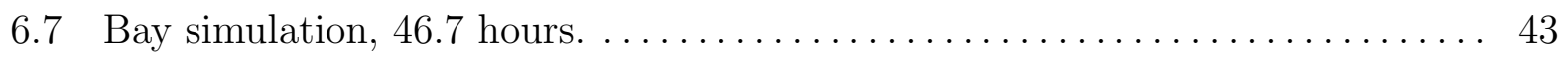

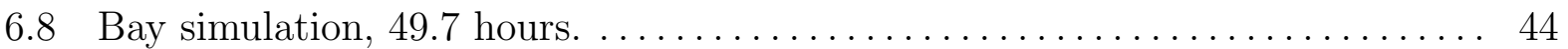

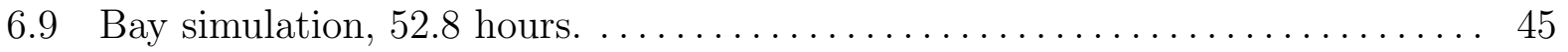




\section{CHAPTER 1}

\section{INTRODUCTION}

The goal of this project is to provide the capability for simulating fluid flow on complicated geometries, such as in the Bahia de Todos Santos. The Bahia de Todos Santos is a bay situated in the northwest corner of Mexico, off the coast of Ensenada and south of San Diego, California, USA. Figure 1.1 shows the Bahia de Todos Santos. It is part of an image taken from the Moderate Resolution Imaging Spectroradiometer (MODIS) sensors on the Aqua and Terra satellites in late June and early July 2003 [8]. Roughly 200 square kilometers in size, the bay also contains two islands off the peninsula of Punta Banda. Characteristics of flow in this bay are driven by the moon tide (M2) and wind forcing [9].

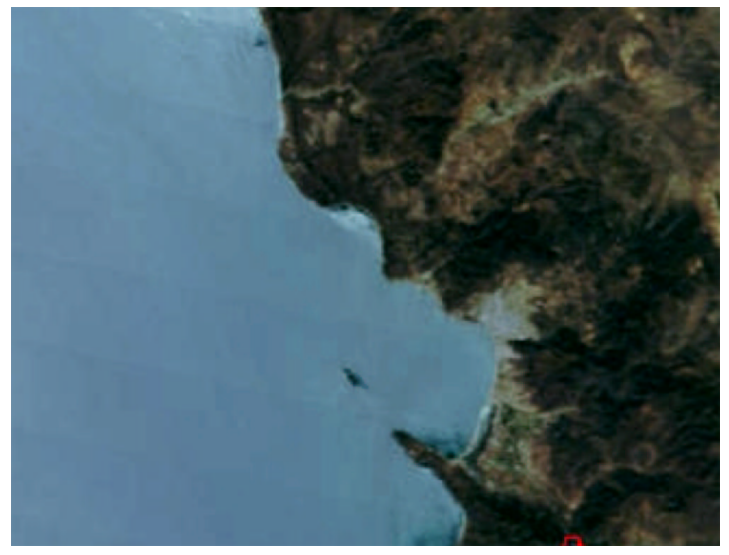

Figure 1.1: Satellite image of the Bahia de Todos Santos. Credit: Jacques Descloitres, MODIS, Rapid Response Team, National Aeronautics and Space Administration (NASA)/Goddard Space Flight Center (GSFC). "Fires in Northern Baja California, Mexico (5 of 7)," NASA Visible Earth Website, 2003. [8]

It is important to understand the fluid dynamics in bays such as the Bahia de Todos Santos because of the wide range of dependencies on the flow such as bay ecosystems, coastal erosion and pollution dispersion [6]. To study any of these in a lab is difficult and expensive; for this reason, computer simulation plays an important role in investigating 
flow phenomena in large bodies of water.

Common to the computing standards of 1991, the previous study on the Bahia de Todos Santos used a relatively coarse geometry on a Cartesian grid, see Figure 1.2 [9]. This grid lacks the accuracy common to today's computing standards to represent the dynamic geometry of flow fields such as the bay. The kilometer-by-kilometer grid cells do not adequately capture the island geometry, since the islands are little bigger than the grid cell.

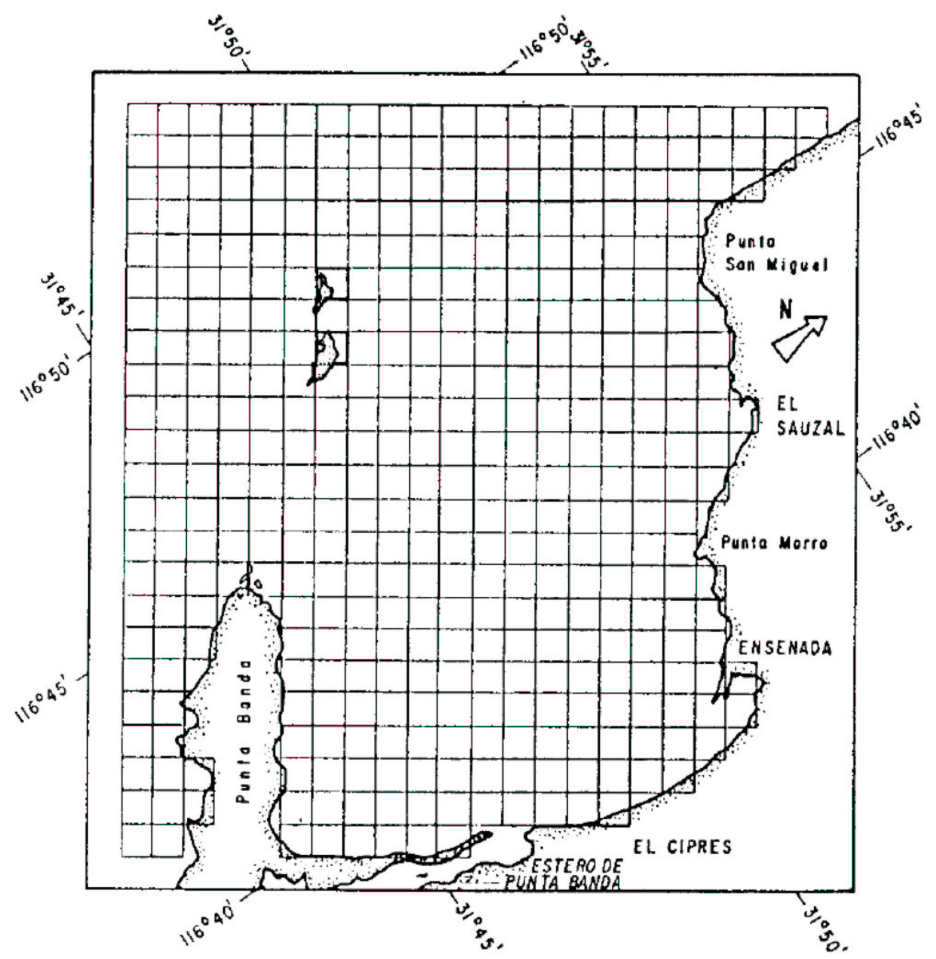

Figure 1.2: Grid used by in previous study of the Bahia de Todos Santos [9]. F.J. Gavidia Medina Espinoza, M.L. Argote and A. Amador Buenrostro. Wind- induced circulation in Todos Santos Bay, B.C., Mexico. Atmosphera, 4:101-115, 1991.

It is the objective of this study to provide a means to more accurately represent the geometry of the bay for simulating the shallow water equations by using curvilinear, overset grids. By transforming the shallow water equations to curvilinear coordinates and using a finite difference scheme, it is possible to simulate this problem on complex geometry. Figure 1.3 shows the transformation from Cartesian to curvilinear coordinates. 


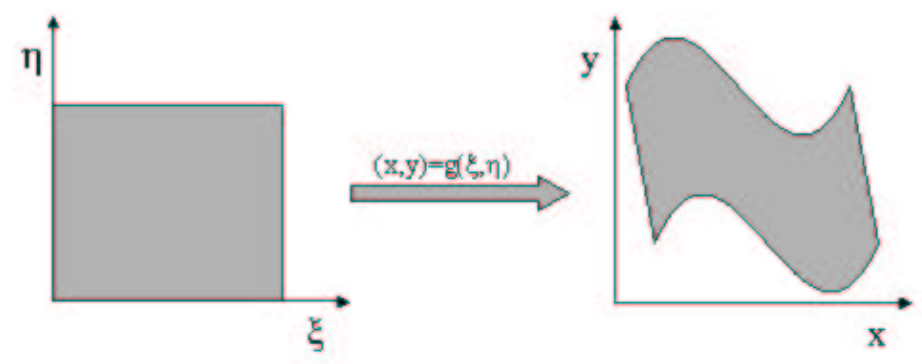

Figure 1.3: Transformation from Cartesian coordinates to curvilinear coordinates.

By using overset grids, the capability of the algorithm is expanded to include complex geometry that is not suitable for a single grid. Overset grids using the Overture software framework easily allows for the inclusion of islands, see Figure 1.4.

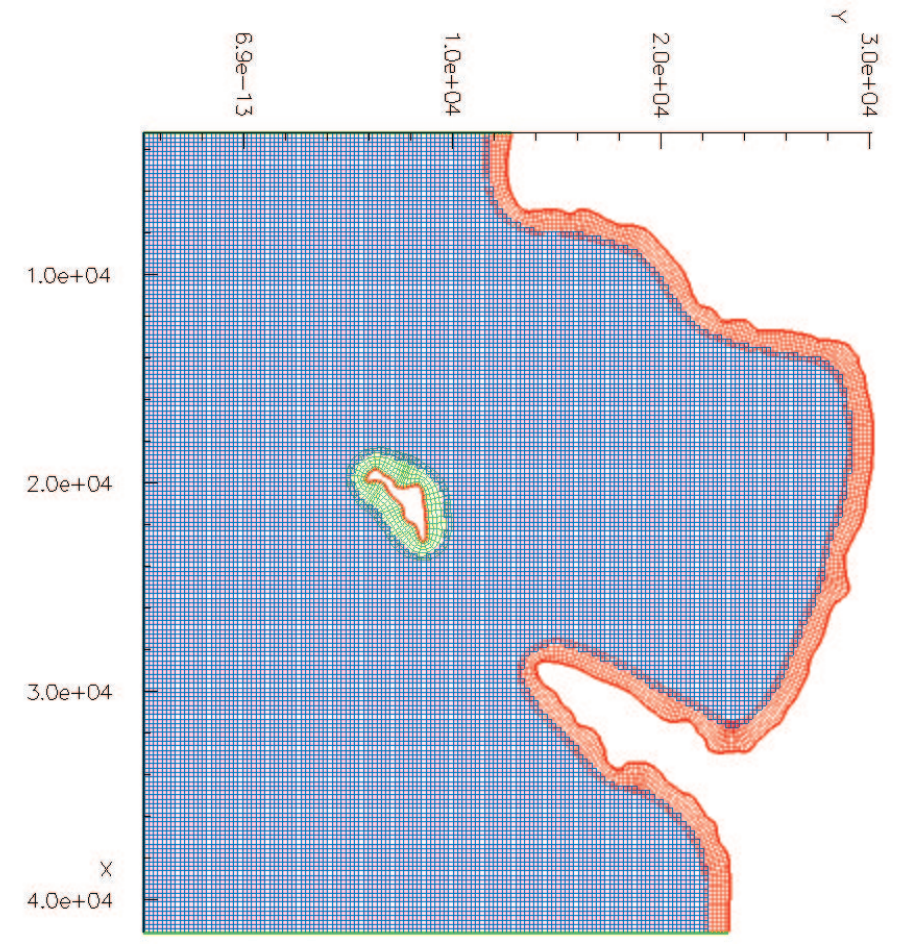

Figure 1.4: Overset grid of the Bahia de Todos Santos. 
It is important to note that this study is focused on expanding the capability of solving the shallow water equations with the bay in mind, rather than an oceanographic focus on solving the particular problems of the bay itself. The examples in this study show the feasibility of the method presented here in solving problems of this nature. The increased accuracy and efficiency of this method over the previous study of the Bahia de Todos Santos lies in the ability to change the geometry without changing the algorithm. Flows near the coastline can be more accurately reproduced with the inclusion of more details in the grid. Though the set up of boundary conditions and other features of each geometry - such as tides, wind, and bottom friction - need to be configured for each particular problem, the solution algorithm is independent of the geometry.

\subsection{Previous Work}

There is a wide range of finite difference studies in the area of shallow water equations available in the literature $[1,2,4,6,12,19,20,22,23,24]$. The governing equations are derived from the equilibrium equations of horizontal forces and the mass continuity equation for incompressible fluid [17]. Leendertse was the first to modify an alternating direction implicit (ADI) scheme to split the two-step method to include an implicit and an explicit stage for each half time step [19]. Ponce used this same ADI method to study recirculation effects in simple channel configurations for Cartesian coordinates [23]. Johnson used the semi-implicit ADI method to solve the nonlinear transformed equations in general coordinates [20]. A staggered grid was used by Scott and Barber, who solved the explicit transformed shallow water equations; though effective stresses were not used for this study, they did employ a wetting and drying scheme [25]. Borthwick and Barber finally applied the modified alternating direction implicit scheme of Leendertse to the complete, nonlinear, transformed shallow water equations [4]. Casulli developed a semiimplicit algorithm for the fully transformed shallow water equations which currently includes both 2-D and 3-D solvers that have been used to model behavior, among other

places, the San Diego and the San Francisco Bays [6]. Current areas of interest in the development of computationally modelling the shallow water equations branches out into 
the study of island wakes $[1,12,22]$, near-shore circulation and wave breaking effects [26], wetting and drying schemes [2] and parallelization [24]. While there are many forms of the equations, this study uses the notation and form of the equations as given by Borthwick and Barber [4].

\subsection{Included in this Study}

The scope of this work includes the introduction of the shallow water equations and notation, as well as the extensions to the shallow water equations such as how the bed friction, eddy viscosity and wind stresses are applied (Chapter 2). The equations of motion are transformed to curvilinear coordinates in Chapter 3. The next chapter, Chapter 4, discusses in detail the discretization implemented and the boundary conditions used for this study. These three chapters are considered an extension to the introduction to the problem before the results are shown. Initially, the curvilinear form of the shallow water equations is implemented on single grids to produce convergence results for verification and run some initial physical tests to explore the limits behavior of the algorithm (Chapter 5). Once verification with the physical tests is established, the algorithm is then expanded to include overset grids using Overture [7]. Results from the simulation

of the Bahia de Todos Santos are presented in Chapter 6. Concluding remarks in Chapter 7 will discuss conclusions and areas needing to be addressed in future work. 


\section{CHAPTER 2}

\section{CARTESIAN EQUATIONS AND PROBLEM DEFINITION}

For the shallow water equations to be physically valid, several assumptions need to be made about the system being modelled. The body of water must be shallow with relatively uniform density, ensuring that layering effects are not prominent in describing the general flow field. The water needs to be well mixed so that upwelling does not significantly affect fluid flow. Further, the average velocity profile in the vertical direction must be, for the most part, uniform so that an averaged velocity will accurately describe the total direction and magnitude of the flow [23]. In these instances (such as a shallow coastal area, river or lake), the shallow water equations can be used to predict behavior. For a presentation of the physical variables used in this document, refer to Figure 2.1.

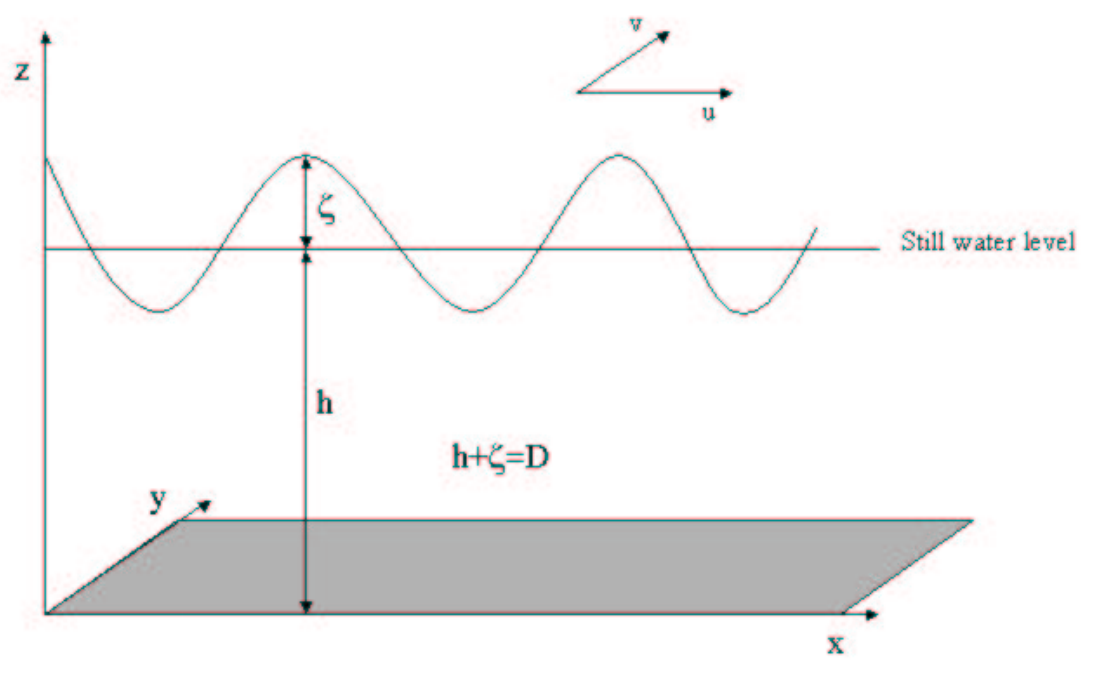

Figure 2.1: Problem definition.

For the test cases leading up to the Bahia de Todos Santos simulation, the physical sizes of the test grids are small enough to make the Coriolis force negligible, and is not included in the code even though Coriolis forces do contribute to the flow [9]. Though 
it is problematic to not include the Coriolis force for the bay simulation, it is considered less of a problem than not including the bottom bathymetry data which also did not get included in the simulation for this thesis project. The depth averaged, shallow water equations are [4],

$$
\begin{gathered}
\frac{\partial \zeta}{\partial t}+\frac{\partial(u D)}{\partial x}+\frac{\partial(v D)}{\partial y}=0 \\
\frac{\partial u}{\partial t}+u \frac{\partial u}{\partial x}+v \frac{\partial u}{\partial y}=f_{c} v-g \frac{\partial \zeta}{\partial x}+\frac{\tau_{w x}-\tau_{b x}}{\rho D}+\frac{1}{\rho D}\left[\frac{\partial\left(D T_{x x}\right)}{\partial x}+\frac{\partial\left(D T_{x y}\right)}{\partial y}\right] \\
\frac{\partial v}{\partial t}+u \frac{\partial v}{\partial x}+v \frac{\partial v}{\partial y}=-f_{c} u-g \frac{\partial \zeta}{\partial y}+\frac{\tau_{w y}-\tau_{b y}}{\rho D}+\frac{1}{\rho D}\left[\frac{\partial\left(D T_{x y}\right)}{\partial x}+\frac{\partial\left(D T_{y y}\right)}{\partial y}\right],
\end{gathered}
$$

where $x$ and $y$ are the Cartesian physical coordinates; $u$ and $v$ are the $x$ and $y$ respective depth averaged velocities; $\zeta$ is the surface elevation; $g$ is gravity; $\tau_{b} x$ and $\tau_{b} y$ are the bed frictions in the $\mathrm{x}$ and $\mathrm{y}$ directions; $\tau_{w} x$ and $\tau_{w} y$ are the wind forcing terms in the $\mathrm{x}$ and $\mathrm{y}$ directions; $\rho$ is water density; $f_{c}$ is the Coriolis parameter; the local water column height is $D=h+\zeta$, where $h$ is the constant depth measured from still water level. $T_{x x}, T_{x y}$, and $T_{y y}$ are the effective shear stresses. Implementing Boussinesq eddy viscosity, the effective shear stresses can be written in the following form [4],

$$
\begin{gathered}
T_{x x}=2 \rho \nu \frac{\partial u}{\partial x} \\
T_{x y}=\rho \nu\left(\frac{\partial u}{\partial y}+\frac{\partial v}{\partial x}\right), \\
T_{y y}=2 \rho \nu \frac{\partial v}{\partial y}
\end{gathered}
$$

where $\nu$ is the eddy viscosity coefficient.

\section{$2.1 \quad$ Bed Friction}

A Chézy expression is used for the bed shear stress components, $\tau_{b x}$ and $\tau_{b y}$, in addi- 
tion to the quadratic friction law (relating bed shear stress to depth averaged velocity):

$$
\begin{aligned}
& \frac{\tau_{b x}}{\rho}=\frac{g}{C^{2}} u\left(u^{2}+v^{2}\right)^{\frac{1}{2}}, \\
& \frac{\tau_{b y}}{\rho}=\frac{g}{C^{2}} v\left(u^{2}+v^{2}\right)^{\frac{1}{2}},
\end{aligned}
$$

where $g$ is gravity, and $C$ is the Chézy coefficient [23].

There are other means of expressing the bed friction such as Manning's equation ,

$$
\begin{aligned}
& \frac{\tau_{b x}}{\rho}=\frac{n^{2} g}{D^{1 / 3}} u\left(u^{2}+v^{2}\right)^{\frac{1}{2}}, \\
& \frac{\tau_{b y}}{\rho}=\frac{n^{2} g}{D^{1 / 3}} v\left(u^{2}+v^{2}\right)^{\frac{1}{2}},
\end{aligned}
$$

where $n$ is the Manning roughness factor [23].

\subsection{Eddy Viscosity}

There are many ways to implement the eddy viscosity terms in the momentum equations [13]. A brief mention of some of these approaches is in order because the simplest method - constant eddy viscosity — will be implemented here, but any future work in this area must take into account a more substantial turbulence model. For turbulence, a one or two equation model is necessary in which $\nu$ is solved at each time step by additional equations (such as the $k-\epsilon$ turbulence models) [13]. There are also simple algebraic relationships that can be implemented to vary the eddy viscosity across the domain depending on the depth and magnitude of the flow.

For this project, constant eddy viscosity was selected because of simplicity. This option, however, is problematic in that it is not well-defined what the value of the coefficient of eddy viscosity, $\nu$, should be for different grids. Historically, a value has been selected on the order of the width of the shear layer and the magnitude of the difference in the velocities $[10,18,27]$. For this study, values for $\nu$ are chosen arbitrarily but with some guidance from theory. Theoretically, $\nu$ is chosen to be the same order as the charac- 
teristic length times the characteristic velocity [3]. The final value is chosen on whether the results seem, or can be proven to be, valid (the best case would be comparing results with field data to select the appropriate values).

Unfortunately, this simple model is not desirable for simulations run on the Bahia de Todos Santos and any further study on this project would demand the inclusion of a more appropriate turbulence model.

\subsection{Wind}

While wind forcing can be used in this study, it is difficult to appropriately model wind where the forcing takes place on an averaged velocity rather than a surface layer of a three dimensional model (or at least some model capable of layering). Despite this drawback, wind is included as a constant across the domain using the following expression $[3]$

$$
\begin{aligned}
& \frac{\tau_{w x}}{\rho}=K|w| w_{x}, \\
& \frac{\tau_{w y}}{\rho}=K|w| w_{y},
\end{aligned}
$$

where $K$ is the wind stress coefficient, $|w|$ is the magnitude of the wind velocity, $w_{x}$ and $w_{y}$ are the components of the wind velocity [3]. Magnitude for the wind stress coefficient and wind direction for the Bahia de Todos Santos is dependent on seasonal conditions and are taken from [9]. 


\section{CHAPTER 3}

\section{TRANSFORMING THE EQUATIONS}

Transforming the equations from the physical coordinate system to a computational domain (see Figure 1.3) is defined as, $x=x(\xi, \eta)$, and $y=y(\xi, \eta)$. To derive the transformation of the derivatives to the computational domain, use the chain rule $[14,16]$. The derivatives become,

$$
\begin{aligned}
\frac{\partial}{\partial x} & =\frac{\partial \xi}{\partial x} \frac{\partial}{\partial \xi}-\frac{\partial \eta}{\partial x} \frac{\partial}{\partial \eta} \\
\frac{\partial}{\partial y} & =\frac{\partial \xi}{\partial y} \frac{\partial}{\partial \xi}-\frac{\partial \eta}{\partial y} \frac{\partial}{\partial \eta}
\end{aligned}
$$

By using the following relationships,

$$
\begin{gathered}
\frac{\partial \xi}{\partial x}=\frac{1}{J} \frac{\partial y}{\partial \eta}, \\
\frac{\partial \xi}{\partial y}=\frac{-1}{J} \frac{\partial x}{\partial \eta} \\
\frac{\partial \eta}{\partial x}=\frac{-1}{J} \frac{\partial y}{\partial \xi} \\
\frac{\partial \eta}{\partial y}=\frac{1}{J} \frac{\partial x}{\partial \xi},
\end{gathered}
$$

where,

$$
J^{-1}=\left(\frac{\partial x}{\partial \xi} \frac{\partial y}{\partial \eta}-\frac{\partial x}{\partial \eta} \frac{\partial y}{\partial \xi}\right),
$$

then the following transformation equations can be used,

$$
\begin{aligned}
& \frac{\partial f}{\partial x}=\frac{1}{J}\left(y_{\eta} \frac{\partial f}{\partial \xi}-y_{\xi} \frac{\partial f}{\partial \eta}\right), \\
& \frac{\partial f}{\partial y}=\frac{1}{J}\left(x_{\xi} \frac{\partial f}{\partial \eta}-x_{\eta} \frac{\partial f}{\partial \xi}\right)
\end{aligned}
$$


where $f$ is a function and $x_{\xi}, x_{\eta}, y_{\xi}$ and $y_{\eta}$ are the metric terms used for the computation. Since the metric terms are determined by the grid and do not change over time, they will be expressed in the simplified derivative form of $x_{\xi}$.

The transformed shallow water equations are,

$$
\begin{gathered}
\frac{\partial \zeta}{\partial t}+\frac{1}{J}\left[y_{\eta} \frac{\partial(u D)}{\partial \xi}-y_{\xi} \frac{\partial(u D)}{\partial \eta}+x_{\xi} \frac{\partial(v D)}{\partial \eta}-x_{\eta} \frac{\partial(v D)}{\partial \xi}\right]=0, \\
\frac{\partial u}{\partial t}+\frac{1}{J}\left(y_{\eta} u \frac{\partial u}{\partial \xi}-y_{\xi} u \frac{\partial u}{\partial \eta}+x_{\xi} v \frac{\partial u}{\partial \eta}-x_{\eta} v \frac{\partial u}{\partial \xi}\right)=f_{c} v-\frac{g}{J}\left(y_{\eta} \frac{\partial \zeta}{\partial \xi}-y_{\xi} \frac{\partial \zeta}{\partial \eta}\right) \\
+\frac{\tau_{w x}-\tau_{b x}}{\rho D}+\frac{1}{\rho D J}\left[y_{\eta} \frac{\partial\left(D T_{x x}\right)}{\partial \xi}-y_{\xi} \frac{\partial\left(D T_{x x}\right)}{\partial \eta}+x_{\xi} \frac{\partial\left(D T_{x y}\right)}{\partial \eta}-x_{\eta} \frac{\partial\left(D T_{x y}\right)}{\partial \xi}\right], \\
\frac{\partial v}{\partial t}+\frac{1}{J}\left(y_{\eta} u \frac{\partial v}{\partial \xi}-y_{\xi} u \frac{\partial v}{\partial \eta}+x_{\xi} v \frac{\partial v}{\partial \eta}-x_{\eta} v \frac{\partial v}{\partial \xi}\right)=-f_{c} u-\frac{g}{J}\left(x_{\xi} \frac{\partial \zeta}{\partial \eta}-x_{\eta} \frac{\partial \zeta}{\partial \xi}\right) \\
+\frac{\tau_{w y}-\tau_{b y}}{\rho D}+\frac{1}{\rho D J}\left[y_{\eta} \frac{\partial\left(D T_{x y}\right)}{\partial \xi}-y_{\xi} \frac{\partial\left(D T_{x y}\right)}{\partial \eta}+x_{\xi} \frac{\partial\left(D T_{y y}\right)}{\partial \eta}-x_{\eta} \frac{\partial\left(D T_{y y}\right)}{\partial \xi}\right],
\end{gathered}
$$

where $\xi$ and $\eta$ are the computational coordinate directions. The transformed shear stress equations are,

$$
\begin{gathered}
T_{x x}=\frac{2 \rho \nu}{J}\left(y_{\eta} \frac{\partial u}{\partial \xi}-y_{\xi} \frac{\partial u}{\partial \eta}\right), \\
T_{x y}=\frac{\rho \nu}{J}\left(x_{\xi} \frac{\partial u}{\partial \eta}-x_{\eta} \frac{\partial u}{\partial \xi}+y_{\eta} \frac{\partial v}{\partial \xi}-y_{\xi} \frac{\partial v}{\partial \eta}\right), \\
T_{y y}=\frac{2 \rho \nu}{J}\left(x_{\xi} \frac{\partial v}{\partial \eta}-x_{\eta} \frac{\partial v}{\partial \xi}\right),
\end{gathered}
$$

where $\nu$ is the eddy viscosity. Furthermore, the friction, wind and Coriolis terms remain the same as the Cartesian equations after the transformation. 


\section{CHAPTER 4}

\section{OVERVIEW OF THE NUMERICAL SCHEME}

The finite difference solution method used for this project is the second-order accurate, explicit, Adams predictor-corrector, time-stepping scheme [11, 14],

$$
\begin{aligned}
& u_{j, k}^{p}=u_{j, k}{ }^{n}+\frac{\Delta t}{2}\left[3\left(L_{u}^{n}\right)_{j, k}-\left(L_{u}^{n-1}\right)_{j, k}\right], \\
& u_{j, k}{ }^{n+1}=u_{j, k}{ }^{n}+\frac{\Delta t}{2}\left[3\left(L_{u}^{p}\right)_{j, k}-\left(L_{u}^{n}\right)_{j, k}\right] .
\end{aligned}
$$

The method consists of two steps, a predictor step and a corrector step. Operator $L$ represents the right hand side of the finite difference equation (see Appendix $\mathrm{C}$ for a definition of operator $L$ ) and $u^{p}$ represents the predictor step. Centered differences are used for all spatial derivatives.

It is appropriate to note that some methods employ a spatial $u$ and $v$ averaging in some instances of the solution in the name of "depth averaging" [3]. This technique, while useful in providing dissipation to the solution and hence stabilizing some solutions that may otherwise be unstable, is not necessary. Indeed, it makes little sense to include spatial averaging to account for depth information. The averaging is already included in the way the equations are derived and hence not necessary to include here.

There are also examples of filtering or averaging routines used in studies that require more stability $[3,23]$. These filters are mainly used to dampen high frequency oscillation errors in surface elevation that probably arise from time step errors and the fact that the advection equation has no diffusive term. In this study, an added numerical diffusivity term is included in the finite difference equation for the advection equation to dampen errors in the solution. This term is used for the simulations done on the idealized island and overset grids. For the idealized island simulation, high frequency oscillation errors appear because of errors arising from the boundary conditions; for the overset grids, oscillation errors occur due to the use of a fully centered grid. 
The numerical diffusivity added to the surface elevation equation is $\alpha \nabla^{2} D$, where $\nabla^{2} D$ is represented with an undivided difference and $\alpha$ is the strength coefficient. This term is added to the right-hand-side of the continuity equation and is approximated as,

$$
\alpha \nabla^{2} D \approx \alpha\left(D_{j+1, k}^{n}-2 D_{j, k}^{n}+D_{j-1, k}^{n}+D_{j, k+1}^{n}-2 D_{j, k}^{n}+D_{j, k-1}^{n}\right)
$$

\subsection{Boundary Conditions}

Boundary conditions for this model are problematic. In theory, for subsonic flow, two characteristics enter the system and one characteristic leaves the system (for open boundaries); this would necessitate that two boundary conditions be set for inflow and one for outflow [15]. In practice, however, this scheme does not work as well for boundaries that are mixed inflow and outflow, such as in a bay where one boundary may handle all in and out flow for the domain and may change over time. For geometries that are relatively simple where inflow and outflow boundaries are definite, it is necessary to stick to the two inflow and one outflow boundary condition set up.

For all of the simulations in this study up to the section on Overture results, strict adherence to the characteristic study is followed. For inflow, one velocity component and the surface elevation are given while the other velocity component is extrapolated. For outflow, the surface elevation is given and both velocity components are extrapolated. Extrapolation techniques are either zero or first order and are applied as the configuration demands. First order extrapolation is used as a non-reflective boundary condition for outflow [4]. For walls, nonslip boundaries are employed; the velocity components on the boundary are zero while the surface elevation is extrapolated.

All test cases fall within the definition of subsonic flow; supersonic flow would require a different set of boundary conditions. The Froude number, magnitude of the velocity

over the wave celerity (wave celerity is $\sqrt{g D}$ ), is output along the boundaries in the program to make sure it is a value less than one. This ensures the flow is subsonic on the boundary. 
For periodic boundaries, such as the boundary where the grid meets on the O-grid (or Annulus grid), ghost lines are employed around the outside of the physical domain where the values exiting the system are read back in as the inflow conditions. The value replacement happens on two different lines, as follows: the inflow ghost line is set to the line just inside the outflow boundary, while the inflow boundary is set to the outflow boundary ("inflow," and "outflow" in this case are merely labels for opposite grid edges, and not an indication of the flow direction). The process is done for $u$ velocity, $v$ velocity and surface elevation.

For special cases of mixed inflow and outflow boundaries, two boundary condition schemes are attempted. The first is that the direction of the flow is tested along the boundary to determine whether it is inflow or outflow. Once this is determined, the appropriate number of boundary conditions are set. This seems to work for most of the boundary except where the flow along curved boundaries (such as the Annulus) reaches a transition from normal to perpendicular flow into the domain. Since a thorough investigation of boundary conditions is beyond the scope of this study, smoothing in the form of artificial viscosity is added to the surface elevation equation to deal with the error that results from this boundary condition.

The other method of dealing with mixed inflow and outflow boundaries is to use zero order extrapolation on those boundaries for all velocity and surface elevation [21]. This extrapolation method is used for the simulations involving the Bahia de Todos Santos grids, except for surface elevation values described by the M2 tide. While these boundary conditions give stable and reasonable results for the simulations presented here, there are issues with mass conservation that should be addressed for longer runs. Investigating these issues requires stability analysis to be done on the boundary; this analysis is outside the scope of this project. 


\section{CHAPTER 5}

\section{NUMERICAL RESULTS}

Two channel configurations are used to verify that the finite difference scheme is solving the model equations correctly. The first configuration studies the propagation of a surface elevation wave as it moves along the direction of flow over time. The problem is set up so that results can be analyzed as a strictly two dimensional problem, ignoring $v$ velocity. Periodic boundaries were used on all sides of the slightly curvilinear channel so that the surface elevation wave does not propagate out of the domain. Convergence studies are done on short simulation time and long simulation time.

Errors are measured using the Richardson resolution study technique for spatial resolution, and time resolution where spatial resolution does not give any useful information. The recipe for a Richardson resolution study used here is three runs where the resolution is doubled each time (twice as many points). Define $U_{n}, U_{2 n}, U_{4 n}$ as the approximated solution on three grids, each getting finer in resolution than the previous $\left(U_{n}\right.$ has the fewest points). Each cover the same spatial area, but $\Delta x$ gets cut in half on the succes-

sive grid. Also define $\bar{U}$ as the exact solution. Assuming one compares only on the nodes of the coarsest grid points, define,

$$
\begin{array}{r}
E_{n}=U_{n}-\bar{U}, \\
E_{2 n}=U_{2 n}-\bar{U}, \\
E_{4 n}=U_{4 n}-\bar{U}, \\
d_{n, 2 n}=E_{n}-E_{2 n}, \\
d_{2 n, 4 n}=E_{2 n}-E_{4 n},
\end{array}
$$

where $E_{n}, E_{2 n}$ and $E_{4 n}$ are the error at each grid point; and $d_{n, 2 n}, d_{2 n, 4 n}$ are the difference in error from one grid to its double resolution grid.

If we plug in the values and reduce the equations for $d$, which can be computed from 
the numerical solution and do not depend on the exact solution, we get,

$$
\begin{gathered}
d_{n, 2 n}=U_{n}-U_{2 n}, \\
d_{2 n, 4 n}=U_{2 n}-U_{4 n} .
\end{gathered}
$$

Defining the rate of convergence of the method as $\left\|\bar{U}-U_{n}\right\| \approx c(\Delta x)^{p}, c$ is a constant and $p$ is the convergence rate. Then, the error can be approximated as,

$$
\begin{array}{r}
E_{n} \approx c n^{p}, \\
E_{2 n} \approx c(2 n)^{p}, \\
E_{4 n} \approx c(4 n)^{p} .
\end{array}
$$

Arranging $d_{n, 2 n}$ and $d_{2 n, 4 n}$ again, we take the ratio of these to get,

$$
\frac{d_{2 n, 4 n}}{d_{n, 2 n}} \approx \frac{c(2 n)^{p}-c(4 n)^{p}}{c n^{p}-c(2 n)^{p}}=2^{p} .
$$

Then, to find the convergence rate $p$,

$$
p=\log _{2}\left(\frac{d_{2 n, 4 n}}{d_{n, 2 n}}\right)=\log _{2}\left(\frac{\left\|U_{2 n}-U_{4 n}\right\|}{\left\|U_{n}-U_{2 n}\right\|}\right) .
$$

Convergence rates for this study use the $L^{\infty}$ norm to measure error. This is done by taking the maximum error across the entire grid and using that as the measure of error.

There are two different time constraints on the system. The standard CFL (CourantFreidrichs-Lewy, also called the Courant condition) restriction for shallow water applies, as well as a constraint that comes from the diffusive part of the momentum equations. Note, $\Delta x_{\min }$ in this case means the smallest grid spacing within $x$ or $y$. The Courant condition and diffusive time constraint are,

$$
\Delta t \leq \frac{\Delta x_{\min }}{\sqrt{g D}}
$$




$$
\Delta t \leq b \frac{\left(\Delta x_{\min }\right)^{2}}{\nu}
$$

where $b$ is some constant. For stability, the Courant number $\left(C_{r}=\sqrt{g D} \frac{\Delta t}{\Delta x}\right)$ must be less than one.

The second verification simulation tests flow through a straight channel with a linear slope on a Cartesian grid. The Chézy friction law describes the steady state solution of channels with this configuration. Flow is allowed to run downhill until it reaches steady state, and convergence results are compared to the exact solution the Chézy friction law describes. Convergence rates are measured similar to the above-described process, only there is an exact solution to compare to instead of spatial refining.

Once the simulations are done to prove convergence, three more test problems are selected to demonstrate the capabilities of the method on more complicated geometries. An arbitrary river geometry is selected to test how the flow will behave on curved geometries that have expansion and contraction of the flow domain; in terms of what this means to the goal of modelling the Bahia de Todos Santos, this gives an indication of how the fluid reacts when the flow must move between the island and the peninsula. Flow past a sea mount is selected to test how the flow develops to smooth changes in bathymetry; in the Bahia de Todos Santos, there are a few places where the bathymetry drops steeply to deeper water. Finally, flow past an idealized island tests the behavior of the flow around a circle shaped island; the Bahia de Todos Santos has an island. These three more physically interesting test cases indicate the kind of behavior expected to contribute to the Bahia de Todos Santos flow and help explore the capabilities of this particular model.

\subsection{Propagation of a Surface Wave}

The first simulation result presented tests the surface elevation wave propagation on a simple curvilinear grid. This simulation is meant to test a propagating wave through the system using a resolution study to check the convergence rates to make sure the finite 
difference scheme is solving the equations correctly. No friction or viscosity is used for this simulation. The flow is directed in the direction of $x$ with a speed of $1 \mathrm{~m} / \mathrm{s}$, while no flow is set for the $y$ direction. Wind and Coriolis forces are ignored. All boundaries are periodic, employing the use of ghost points outside the physical domain. Figure 5.1 shows the curvilinear grid used for this simulation.

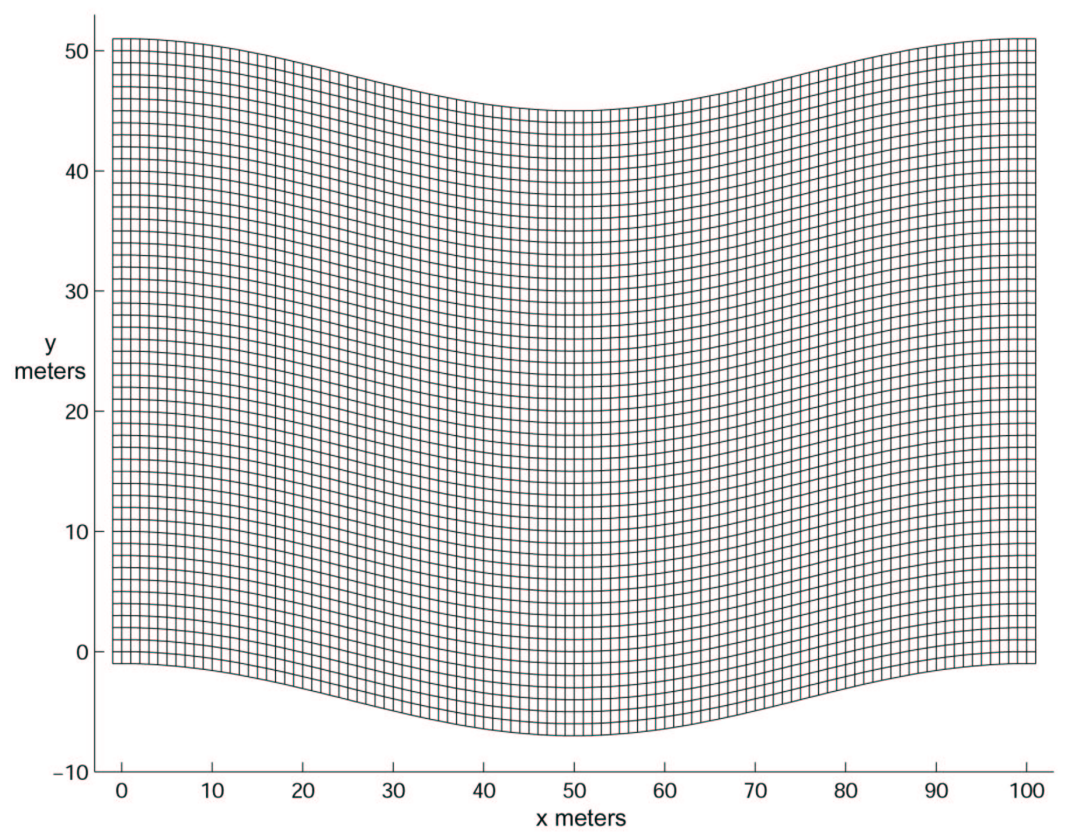

Figure 5.1: Curvilinear grid used for verification study, 100 x 50 meters.

The initial surface elevation is set to a sine wave of 0.05 meters in height. The wave oscillates due to gravity in addition to propagating through the fluid with the momentum. The bottom bathymetry is flat (meaning no variation in depth from the still water level) and is set to 10 meters.

The grid is generated using a standard cosine, deviating slightly from the straight channel flow of the Cartesian case. The physical boundary measurement of the channel is 100 meters by 50 meters, employing 101 and 51 grid points. An extra grid point is generated outside this physical boundary for the ghost point used for the periodic boundary conditions. 
The solution algorithm is in both $x$ and $y$ directions, but results are presented as $x-z$ solution profile pictures for simplicity. Even though the solution algorithm solves across the entire domain, the $x-z$ profile results are the same due to the set up of the problem.

\subsubsection{Short Time Run Results}

As is evident by Figure 5.2, the surface elevation propagates through the domain with the momentum. A time period is used that is consistent with the oscillation of the surface elevation being minimal to see the forward movement of the wave. For the short simulation time of 10 seconds, the wave has propagated forward 10 meters with no apparent distortion and only slight dissipation.

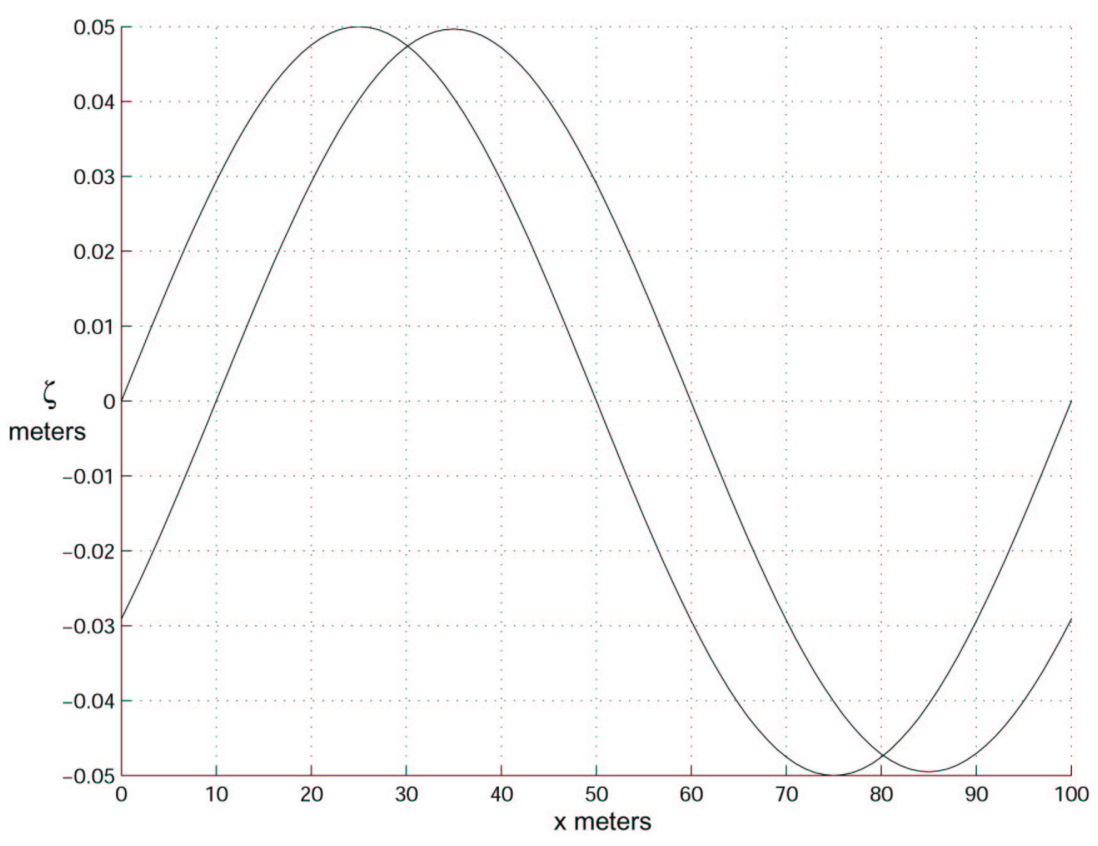

Figure 5.2: Surface elevation profile plot for initial wave and after 10 seconds.

The results show the second order convergence rate one expects from this method, see Table 5.1. The results also show that the round-off error present is maintained at the double precision of the machine used in the code. Additionally, the plots of the error analysis across the grid show that the error is smooth except for the places where the 
error decreases to the accuracy of the machine. Results of the error and convergence rate of the resolution study are taken as the maximum of the differenced values between the coarser grid and one of higher resolution. Values are taken at the node points of the grid with lower resolution. Error in the $v$ momentum equation is not included in the table because it is on the order of $10^{-15}$; since that is the precision of the machine this value is considered zero and therefore the convergence rate makes no sense.

Table 5.1: Short Time Simulation Convergence Study

\begin{tabular}{lrr}
\hline Grids & U error & $\zeta$ error \\
\hline \hline $101 \times 51,201 \times 101$ & $3.14753 \mathrm{e}-04$ & $7.64931 \mathrm{e}-05$ \\
$201 \times 101,401 \times 201$ & $7.87491 \mathrm{e}-05$ & $1.88934 \mathrm{e}-05$ \\
\hline Convergence Rate & 1.99888 & 2.01745
\end{tabular}

\subsubsection{Long Time Run Results}

This test indicates the ability to successfully run the simulation for long periods of time; long simulation times are important to allow the simulation time to reach equilibrium. The simulation is allowed to run so that the surface elevation wave propagates out of the system, and, due to the periodic boundary conditions, return to its original position. The simulation runs for 100 seconds to return to the original position. The convergence rates presented in Table 5.2 indicate that rates are slightly under second order, but are still acceptable.

Table 5.2: Long Time Simulation Convergence Study

\begin{tabular}{lrr}
\hline Grids & U error & $\zeta$ error \\
\hline \hline $101 \times 51,201 \times 101$ & $2.00991 \mathrm{e}-03$ & $1.24931 \mathrm{e}-03$ \\
$201 \times 101,401 \times 201$ & $5.14955 \mathrm{e}-04$ & $3.24580 \mathrm{e}-04$ \\
\hline Convergence Rate & 1.94449 & 1.96461
\end{tabular}


Dissipation in the momentum equations causes the distortion of the wave due to changes in velocity that vary from changes in surface elevation, as seen in Figure 5.3. Solving the surface elevation equation alone, without a change in momentum, causes no distortion of the wave and it propagates perfectly. The distortion causes one part of the wave to steepen, and the other part of the wave to elongate. Diffusion will eventually cause the wave to dissipate to a steady, still water level.

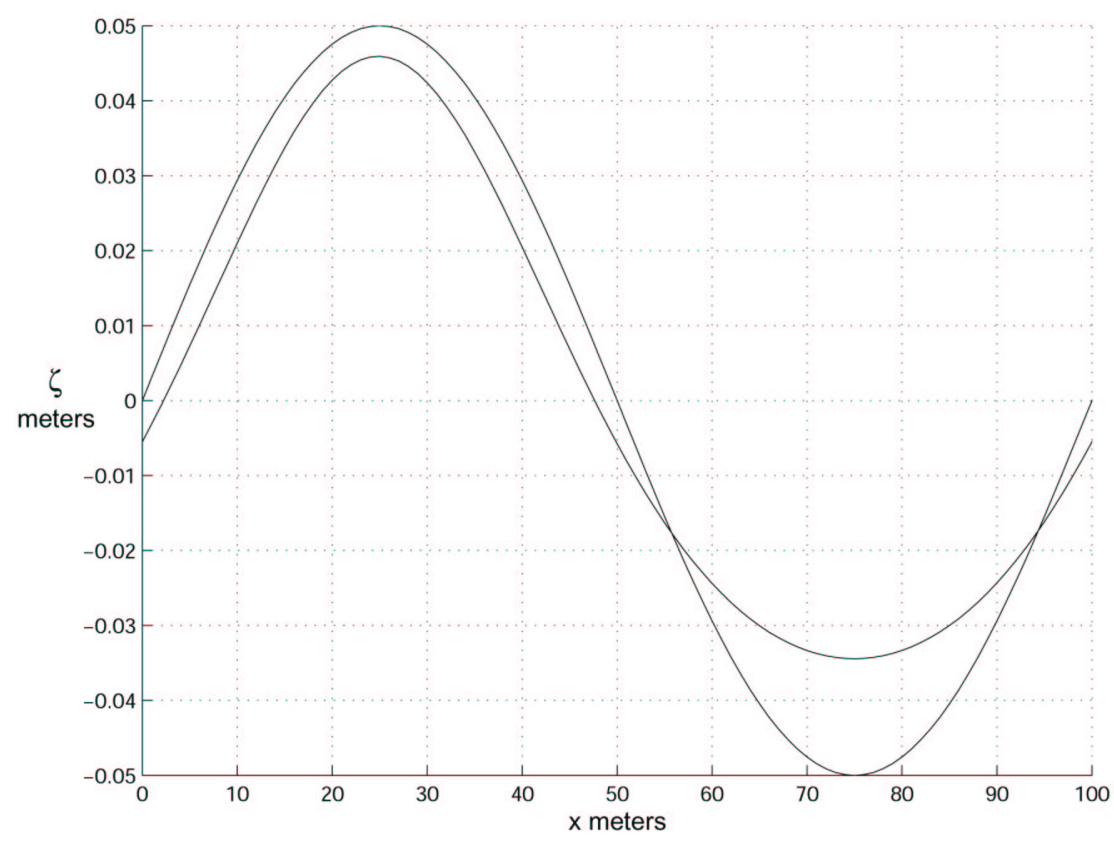

Figure 5.3: Surface elevation profile plot for initial surface wave, and after 100 seconds.

\subsection{Chézy Friction Law}

The Chézy friction law can be used to predict smooth, straight channel flow. It assumes that the surface elevation is constant with the bed slope (i.e. constant depth), and both are linear. The Chézy friction law is derived from assuming the momentum has reached a steady state flow, and that the depth is constant across the field $\left(D_{0}\right)$. Taking the height as some constant plus a slope in the direction of the flow in the $x$ direction, 
and assuming no flow in the $y$ direction, the problem can be reduced to the following,

$$
\begin{gathered}
\partial_{t} \zeta+u \partial_{x} D+D \partial_{x} u=0 \\
\partial_{t} u+u \partial_{x} u=g \partial_{x} \zeta-\frac{g}{D C^{2}} u \sqrt{u^{2}}
\end{gathered}
$$

Because we are assuming a steady state and constant depth, $u \partial_{x} u, u \partial_{x} D, D \partial_{x} u$ and $\partial_{t} u$ are zero. Further, $\partial_{x} \zeta=S$, where $S$ is the preset linear slope. Assume $u>0$.

Since the continuity equation reduces to $\partial_{t} \zeta=0$, the equations reduce to,

$$
u=C \sqrt{S D_{0}}
$$

Using this guide to predict the flow in a straight channel with linear slope and constant depth, we can compare the results to the simulation for verification. Figure 5.4 shows the Cartesian 100 meter by 50 meter grid used for this simulation.

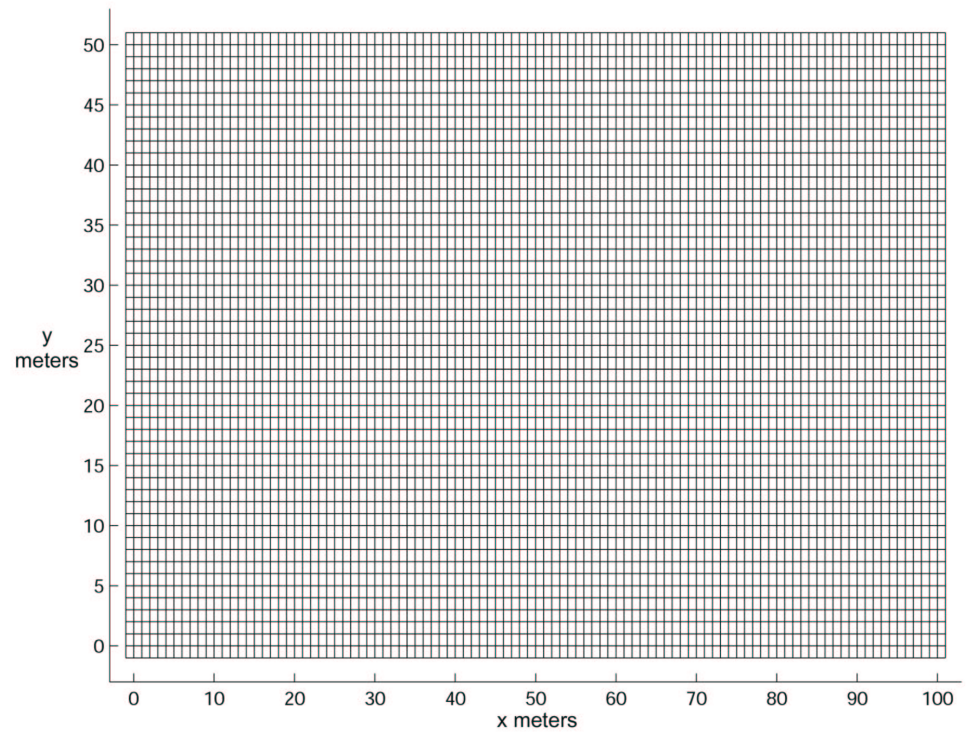

Figure 5.4: Cartesian square grid, 100 meters by 50 meters.

The bottom slope, $S$, is set at 0.001 in the direction of $x$, the total depth is constant at 10 meters, and no variation in the depth in the $y$ direction. The eddy viscosity 
coefficient $\nu$ is set to $0.5 \mathrm{~m}^{2} / \mathrm{s}$. Water density is $1000 \mathrm{~kg} / \mathrm{m}^{3}$. The Chézy roughness coefficient is $41.42 \mathrm{~m}^{1 / 2} / \mathrm{s}$. The time step is 0.05 seconds.

Table 5.3 presents the convergence results as the simulation runs for 500, 1000, 1500 and 2000 seconds. The convergence rate for this problem shows convergence well above second order, which is better than expected for this problem. Since the channel configuration is presented on a Cartesian grid and the surface elevation is initialized to a steady state solution (linear surface elevation slope matching the bottom slope), the solution is exact in the spatial derivatives. Therefore, this example tests the convergence rate for the time step method only and gives a great indication that this part of the application accurately represents the equations.

Table 5.3: Chézy Friction Law Convergence Study

\begin{tabular}{lrc}
\hline Time & $U$ error & Convergence Rate $2^{p}$ \\
\hline \hline 500 seconds & $1.24231 \mathrm{e}-01$ & 3.38754 \\
1000 seconds & $1.18707 \mathrm{e}-02$ & 3.40537 \\
1500 seconds & $1.12036 \mathrm{e}-03$ & 3.40699 \\
2000 seconds & $1.05621 \mathrm{e}-04$ &
\end{tabular}

\subsection{Flow over a Seamount}

Moving on past the simple channel examples for verification, this next simulation tests some of the physical capabilities of the method. This example sets up a seamount in the middle of a straight channel with flow in the $x$ direction. Apart from the general testing of the effect of bathymetry on flow, this simulation also is an exercise in the time series development of the flow and how it logically unfolds as it goes from the initial state to the steady state.

The square Cartesian grid that used for the Chézy friction law example is used again for this simulation, and a seamount is inserted into the bathymetry at the center of the grid. This grid is shown in Figure 5.5. The total depth of the fluid flow is 10 meters, with 
the height of the underwater peak being 2 meters. A slope of 0.001 was used to direct the flow downhill in the direction of $x$, and no variation in the depth in the $y$ direction. The velocity was initialized at $2 \mathrm{~m} / \mathrm{s}$ in the $x$ direction. The eddy viscosity coefficient $\nu$ was chosen to be $1 \mathrm{~m}^{2} / \mathrm{s}$. Water density is $1000 \mathrm{~kg} / \mathrm{m}^{3}$. The Chézy roughness coefficient is set to $41.42 \mathrm{~m} / 1 / 2 / \mathrm{s}$. The time step is 0.02 seconds.

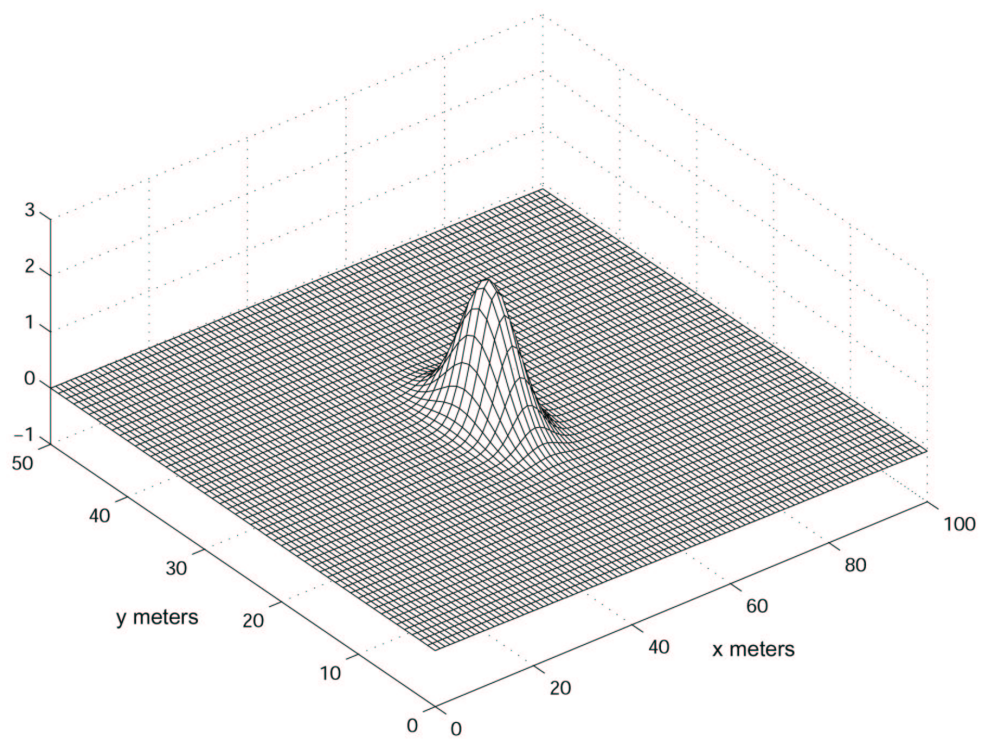

Figure 5.5: Bottom bathymetry and grid for flow past a seamount, $100 \times 50$ meters. The seamount is 2 meters in height.

Normal inflow and outflow boundary conditions are used. For inflow, $v$ velocities and surface elevation is set while $u$ is extrapolated. For outflow, surface elevation is set while $u$ and $v$ are extrapolated. For the outflow extrapolation, first order extrapolation is used as a non-reflective boundary condition. Nonslip boundaries are used along the sides of the channel where $u$ and $v$ are set to zero and surface elevation is extrapolated from interior values.

The velocity and surface elevation behave as expected, see Figure 5.6. Velocity $u$ slows down ahead of the seamount, $v$ diverts flow slightly around the sides of the seamount, and the surface elevation rises ahead of the seamount and drops after the seamount. 

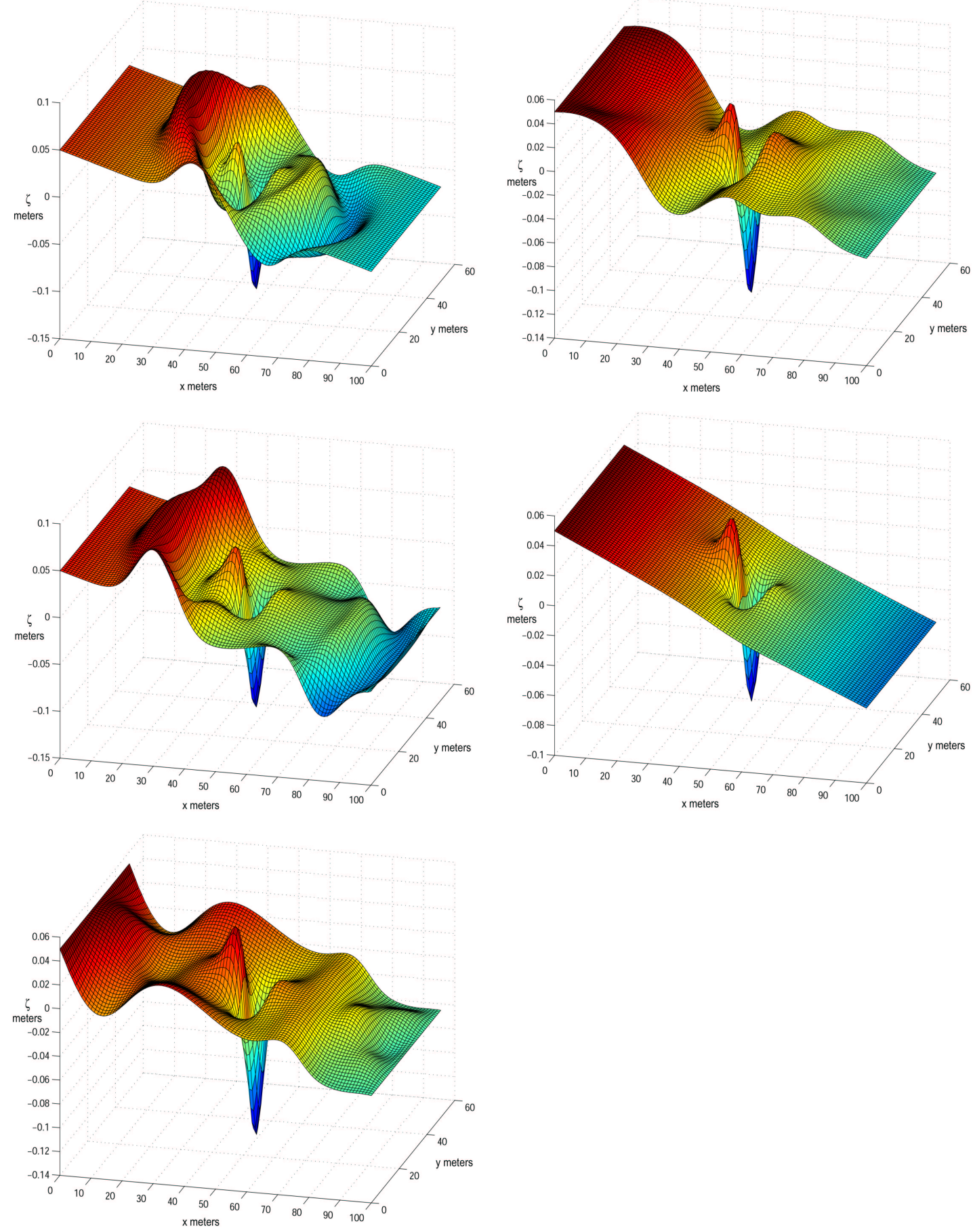

Figure 5.6: Time development of the surface elevation for flow over a seamount. Left column from top: simulation time 2.4, 3.6, and 8 seconds. Right column from top: simulation time 10, and 40 seconds. 
The height of the peak underwater is 2 meters, which, in a 10 meter deep channel changes the surface elevation only slightly. Increasing the slope causes an increase in the magnitude of the perturbations of the surface elevation, and there is a point at which the slope at the seamount causes a shock to the system (the Froude numbers become larger than one). This application is not a study in shock, so results of this nature are not shown here. But it is worth mentioning that if bottom bathymetry can create a situation of shock if the slopes of the bathymetry are steep enough and the flow is initialized to run into it.

The flow is initialized to $2 \mathrm{~m} / \mathrm{s}$ in the $x$ direction, downhill. The fluid builds up on top of the peak initially until gravity forces the flow back down. A resultant wave propagates through the system, both downstream (in the direction of the flow) and upstream (against the direction of the flow). It is acceptable behavior for subsonic flow to allow waves to propagate upstream, and this particular wave is two orders of magnitude below compared to the total depth and does not act as a shock to the system. Once the wave leaves the system upstream and downstream (upstream wave leaves the system after the downstream wave), the system settles down into equilibrium flow where there is a slight alteration in the flow pattern around the hill. Surface elevation has a slight build up of fluid upstream of the bump, and a slight dip in elevation after it. Velocity $u$ slows down ahead of the bottom obstruction, speeds up on top of it and slows down again after passing the seamount. Velocity $v$ also does as expected by diverting flow around the seamount, though the magnitude is small and from a vector standpoint does not significantly alter the main direction of the flow.

Figure 5.6 shows the surface elevation as it develops from the initial state to the steady state. Figure 5.6 at 2.4 seconds shows the initial reaction of the flow; flow is uniform initially, so it builds up initially over the seamount and immediately falls down to produce a wave. Figure 5.6 at 3.6 seconds shows the wave propagating both upstream and downstream where upstream wave propagates slower than the downstream wave. Upstream wave propagation is valid for subsonic flow and indicates agreement with the physics. The waves then propagate out of the system, shown in Figure 5.6 for 8 seconds. 
The surface elevation oscillates after the waves leave the system in a diminished way (Figure 5.6 at 10 seconds) until the system arrives at a steady state (Figure 5.6 at 40 seconds and beyond).

\subsection{Generic River Geometry}

Flow in a river channel is a traditional test case, and is excellent for testing the behavior of the simulation on geometry that has expansion and contraction of the flow domain. The simulation is set up with $x$ direction flow, and nonslip boundaries along the sides of the channel. For the inflow boundary condition, surface elevation and $v$ velocity are set. For the outflow, only the surface elevation is set. Along the nonslip boundaries, $u$ and $v$ are set to zero. Values not set on the boundary are extrapolated, zero order for everywhere except the outflow which is first order.

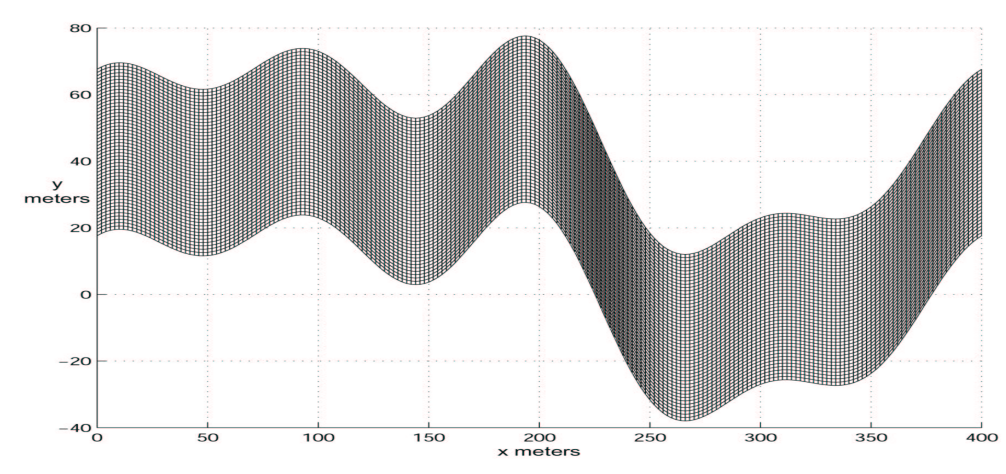

Figure 5.7: Generic river geometry grid, approximately 400 x 50 meters.

To drive the flow, a slope of 0.001 was added in the $x$ direction, and the channel is 8 meters deep. Chézy friction again is set to $41.42 \mathrm{~m}^{1 / 2} / \mathrm{s}$. The eddy viscosity coefficient $\nu$ is $6 \mathrm{~m}^{2} / \mathrm{s}$. Velocities are initialized as 2 meters per second for $u$, and the system is allowed to run until a steady state is reached. Figure 5.7 shows the grid for this simulation. The channel is approximately 50 meters by 400 meters with grid spacing approximately 1 meters. Figure 5.8 shows the steady state simulation results. 

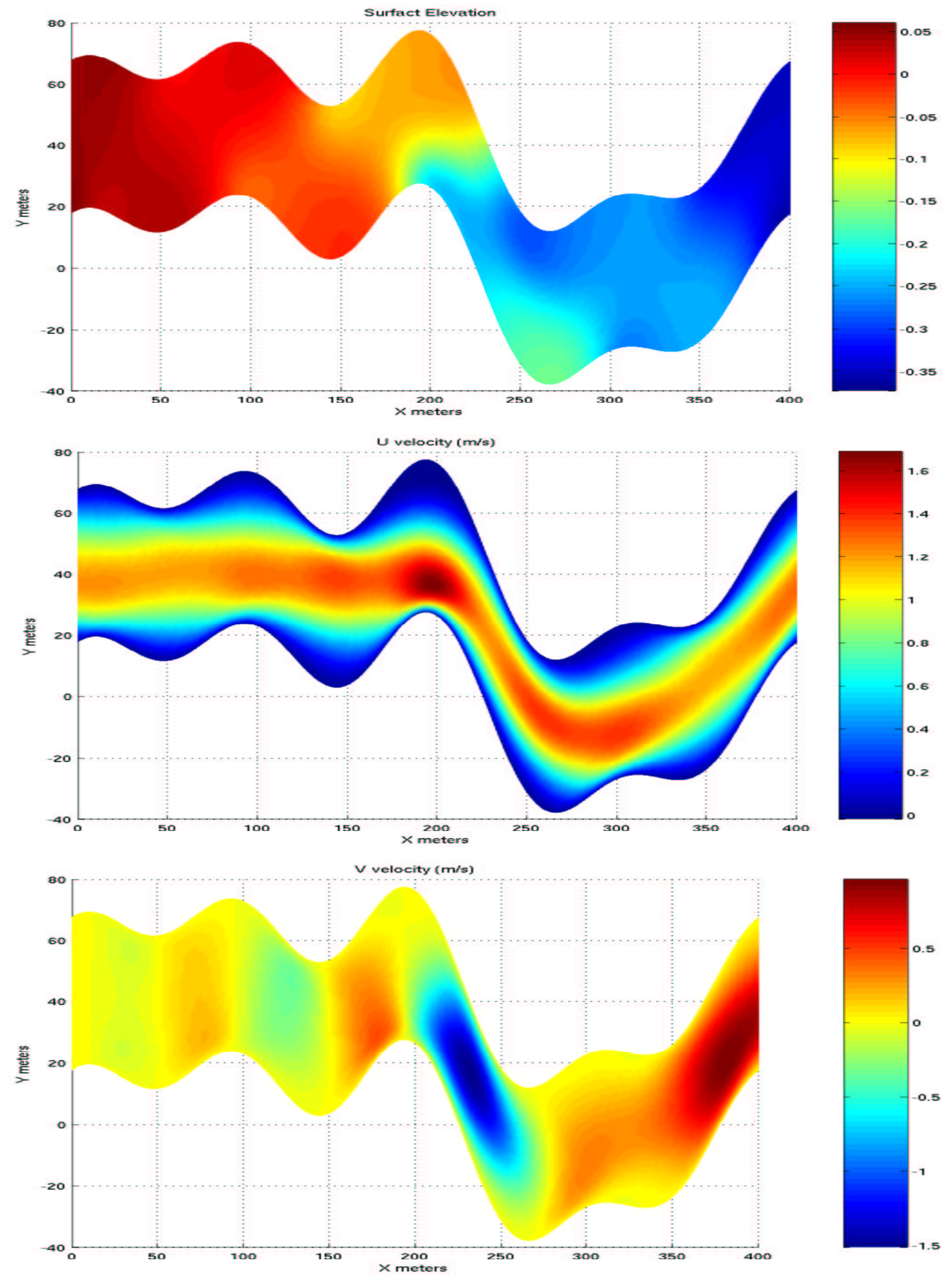

Figure 5.8: From the top: surface elevation, $u$ velocity and $v$ velocity for generic river geometry at steady state. 
The simulation results presented in Figure 5.8 are for the simulation time of 500 seconds. Results indicate agreement with what one would expect. Velocities for $u$ create a higher velocity channel in the center of the geometry, which does not wiggle with small channel changes, but follows the straightest path. Nonslip boundary conditions along the sides of the channel create this channel flow pattern. Velocities for $v$ also behave as expected, diverting flow in the direction of the channel as it curves.

Surface elevation is consistent with the flow pattern. For the slight changes in channel direction, the surface elevation behaves like the straight channel flow where the surface elevation has a linear slope consistent with the bottom slope of the bathymetry. In places where the channel turns sharply, surface elevation is raised and lowered depending on the flow: where the flow undergoes a change of direction, the elevation bulges to accommodate the change.

\subsection{Flow Past an Idealized Island}

Flow past an idealized island is an important test case on the way to exploring real bathymetry of the Bahia de Todos Santos. The set up for this simulation run is an annulus grid where the ends of the grid meet are periodic, the inner boundary is a nonslip wall and the outer boundary is an inflow/outflow mixed boundary. Figure 5.9 shows the annulus grid. The mixed boundary uses the direction of the normal flow to determine whether the flow is coming into or leaving the system. Once the direction of the flow is determined, the appropriate boundary condition is assigned: inflow assigns $u$ velocity component and the surface elevation, outflow only assigns the surface elevation. Values that are not assigned are extrapolated from interior values like previous simulations.

Flow is in the $x$ direction, initialized at $3 \mathrm{~m} / \mathrm{s}$ and no $y$ direction velocity. A gentle slope of 0.001 is in the $x$ direction to help drive the flow. Chézy friction for smooth channel flow is used at $41.42 \mathrm{~m}^{1 / 2}$ per second, water density at $1000 \mathrm{Kg} / \mathrm{m}^{3}$, depth at 25 meters across the domain. Simulations are run for 20,000 seconds. 


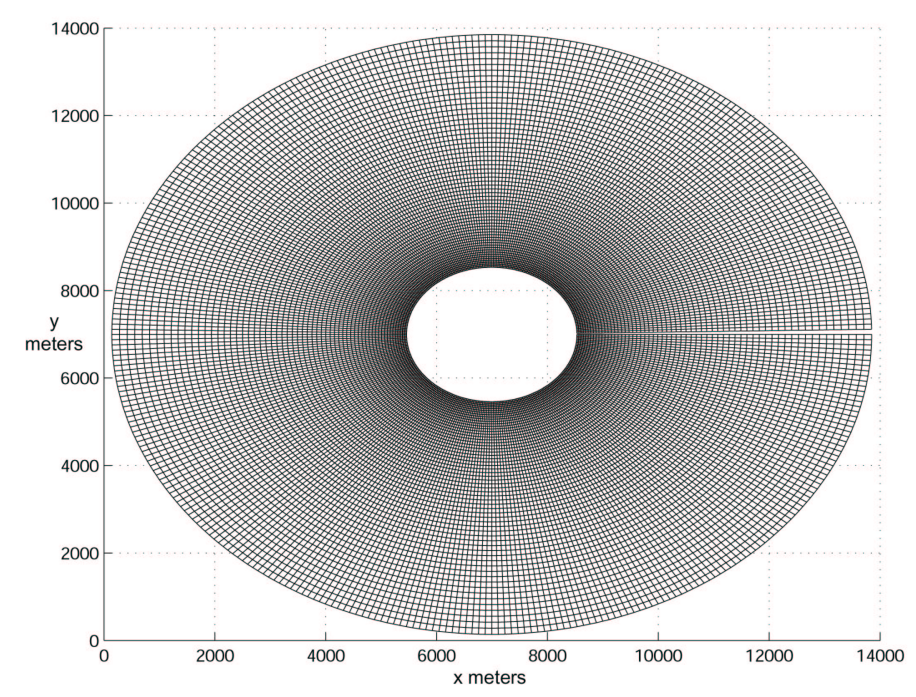

Figure 5.9: Annulus grid for flow past an idealized island.

Artificial viscosity in the continuity equation is to control some error on the outer boundary due to insufficient boundary conditions. Error is generated when the direction of the incoming flow transitions between inflow and outflow (inflow moves from normal to parallel to the boundary).

Simulation results reveal an excellent vortex formation. The formation of the vortices behind the island is controlled by the viscosity; the size and general shape of the vortices can be changed by changing the value of the viscosity coefficient. It is unclear whether this behavior has the capability to demonstrate vortex shedding. To explore this, simulations would need been to be run for long periods of simulation time to allow the vortices to begin oscillating.

The results show, in Figures 5.10 through 5.13, separate runs comparing different eddy viscosity coefficient values. The first run uses the value of $20 \mathrm{~m}^{2} / \mathrm{s}$ and produces a large recirculation area with the large eddy viscosity coefficient value. The second, third and fourth runs at $5 \mathrm{~m}^{2} / \mathrm{s}, 1 \mathrm{~m}^{2} / \mathrm{s}$ and $0.5 \mathrm{~m}^{2} / \mathrm{s}$ coefficients show a reduction in the recirculation field. The results show the circulation pattern behind the island as it changes with the eddy viscosity coefficient, and is presented by the velocity and by the vorticity ( $z$ component of curl). The behavior of the varying eddy viscosity coefficient is important because the value is arbitrarily set. As seen in this example run, the effect of 
the value of the eddy viscosity coefficient has a distinct effect on the flow pattern and it is necessary, for valid scientific simulations using this model, to use an appropriate value (for further discussion on this topic, see Section 2.2). 

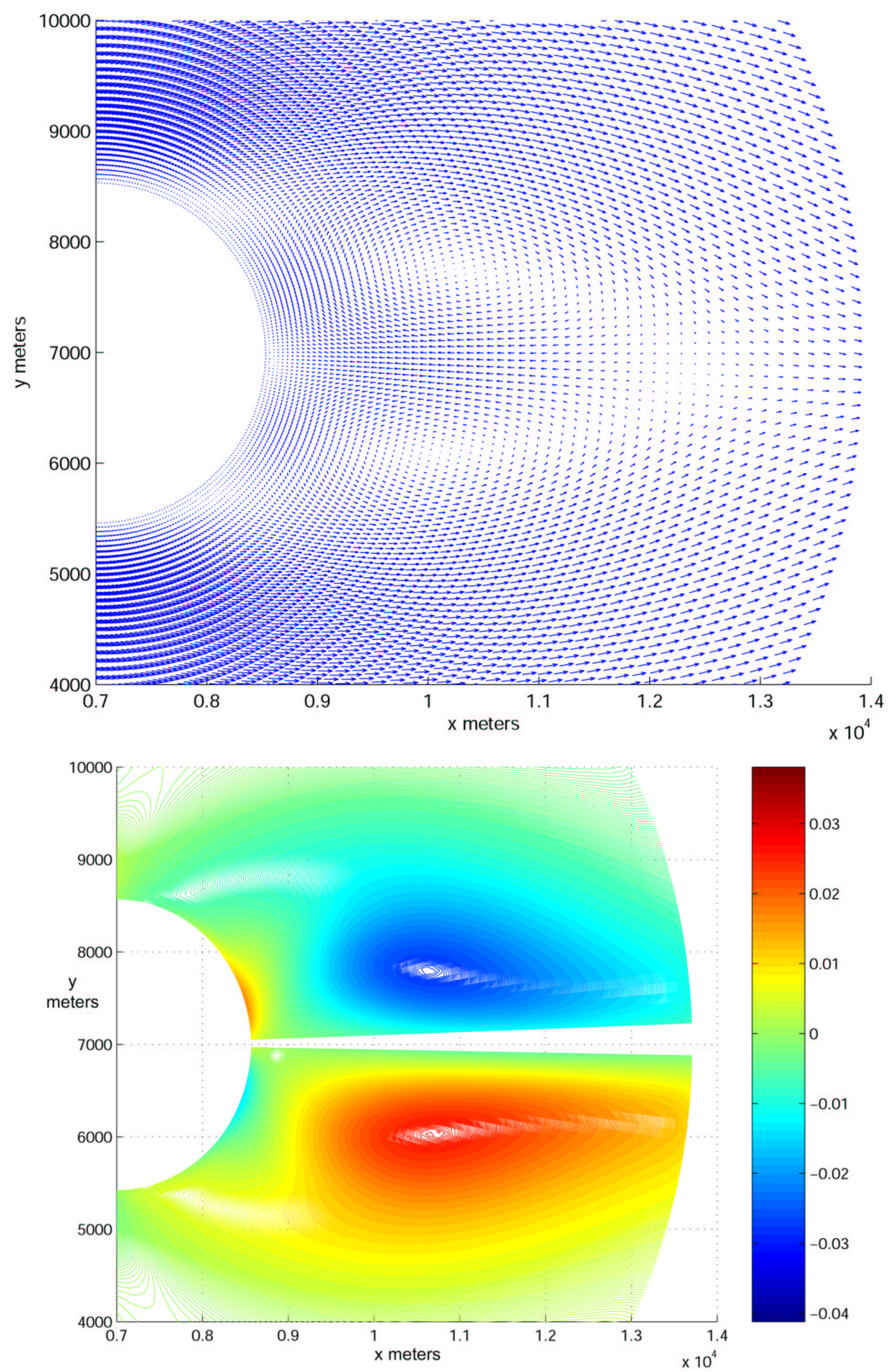

Figure 5.10: Velocity (top) and vorticity (bottom) plots for flow past an idealized island for eddy viscosity $20 \mathrm{~m}^{2} / \mathrm{s}$. These figures indicate the large circulation region associated with the high eddy viscosity. 


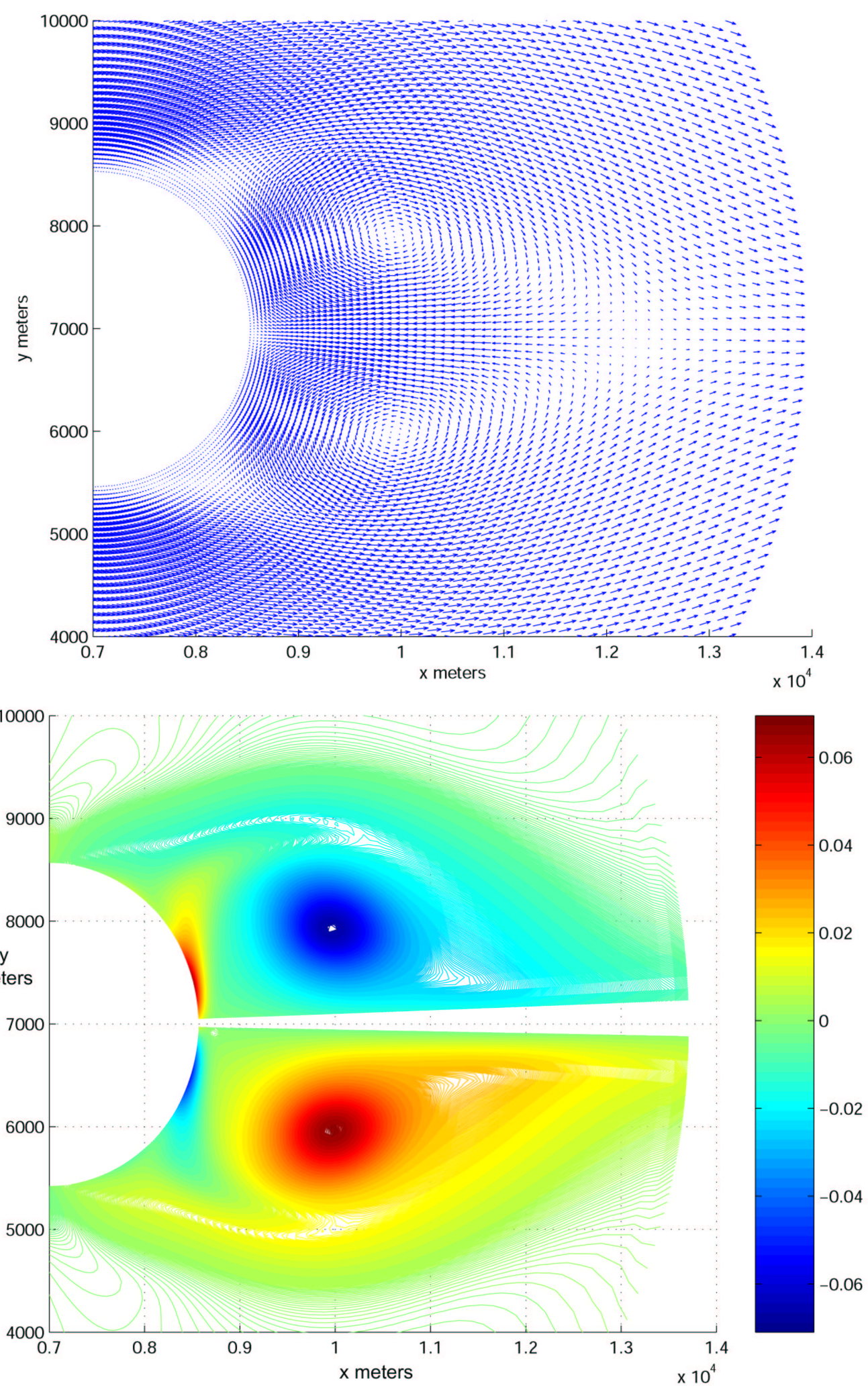

Figure 5.11: Velocity (top) and vorticity (bottom) plots for flow past an idealized island for eddy viscosity $5 \mathrm{~m}^{2} / \mathrm{s}$. These figures indicate a reduction in the circulation region. 

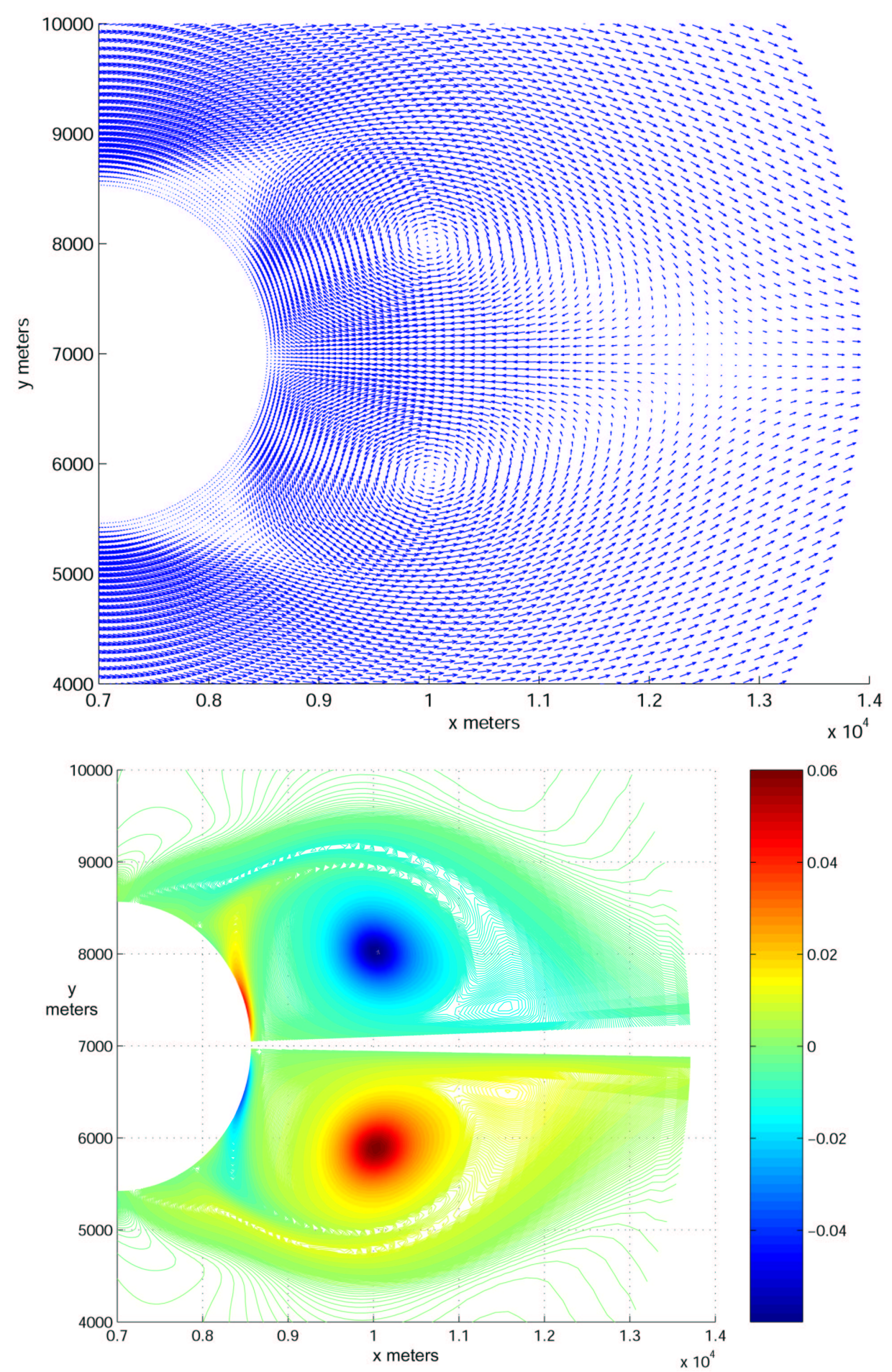

Figure 5.12: Velocity (top) and vorticity (bottom) plots for flow past an idealized island for eddy viscosity $1 \mathrm{~m}^{2} / \mathrm{s}$. These figures indicate a much smaller circulation region for the smaller eddy viscosity. 

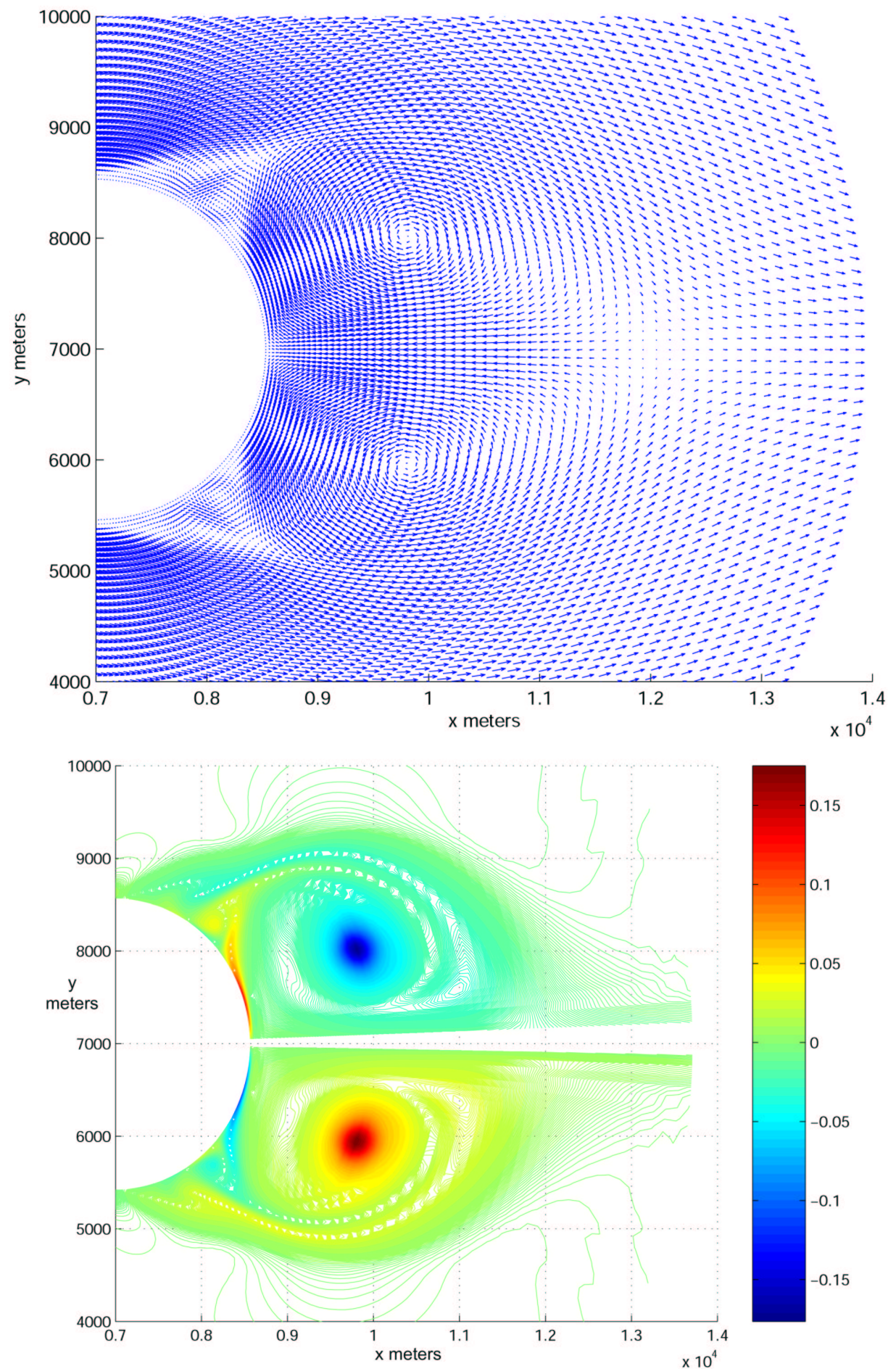

Figure 5.13: Velocity (top) and vorticity (bottom) plots for flow past an idealized island for eddy viscosity $0.5 \mathrm{~m}^{2} / \mathrm{s}$. These figures exhibit some additional vortex formation close to the island. 


\section{CHAPTER 6}

\section{OVERTURE AND THE BAHIA DE TODOS SANTOS}

Overture is a $\mathrm{C}++$ software framework developed at Lawrence Livermore National Laboratory $[5,7]$. It includes tools for developing grids, plotting graphics, and a development library to increase the feasibility of developing large scale overset grid solvers. It is written mostly in $\mathrm{C} / \mathrm{C}++$, and for this study, Overture and its components are compiled and run on a Linux-Pentium workstation. Further description of Overture and directions on installing the framework are located on the web at http://www.llnl.gov/casc/Overture/.

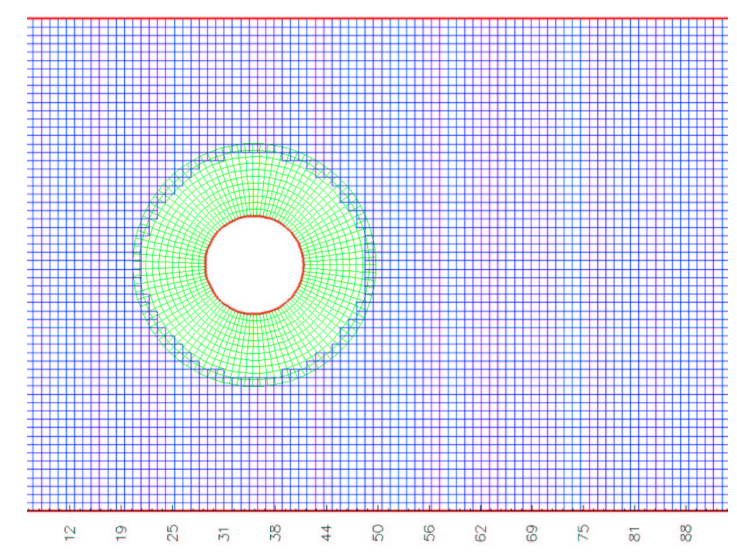

Figure 6.1: Overset grid example, an annulus in a straight channel.

The Overture framework is designed to handle multiple grids and their interaction automatically. The interpolation of data from one grid to another uses biquadratic interpolation (a three by three stencil), which allows second order convergence for second order differential equations [7]. The generated grid file, generated using Ogen (Overture's grid generator), contains the information Overture uses to determine size and interaction of grids. Native Overture operators can be used, if desired, to handle the differencing terms (i.e. the function $u . x()$ takes the $x$ derivative of $u$ and the default is second order, centered difference). Metric terms are also automatically taken into account. Therefore, 
the Cartesian form of the equations can be programmed, further simplifying coding.

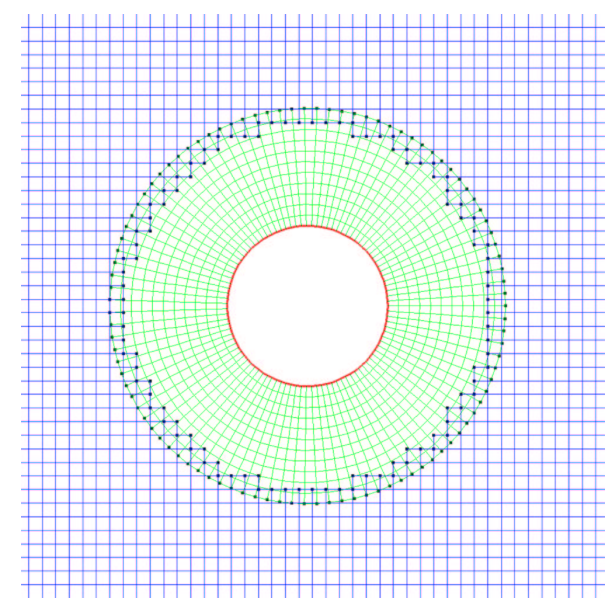

Figure 6.2: Close up of the overset grid, including interpolation points.

To simulate the Bahia de Todos Santos, a version of the Adams predictor corrector method previously described in this study is re-implemented in Overture using the operators provided, and the Cartesian form of the equations. Artificial viscosity is again implemented to help smooth errors that arise from a fully centered grid scheme.

The Bahia de Todos Santos grid is generated using Ogen. Data points were collected from the bathymetry data file using a Matlab script that outlines the coast and island. These data points were placed into an Ogen command script to generate splines from the points that were then used to create the final grid. Three sub-grids have been generated to cover the bay: one curvilinear grid to go around the island, one curvilinear grid to cover the coastline, and one large Cartesian grid to cover the rest of the bay. The bathymetry depth information was not used other than to generate the coastline, instead a constant depth is used across the bay.

Figure 6.3 shows the full grid representation of the Bahia de Todos Santos, created with Ogen. Resolution on the grid is on average 200 by 200 meters per grid cell, with variations for smaller and larger grid cells near the coastline. There are 24,836 grid cells on the grid, and 968 interpolation points for the grid overlapping. 


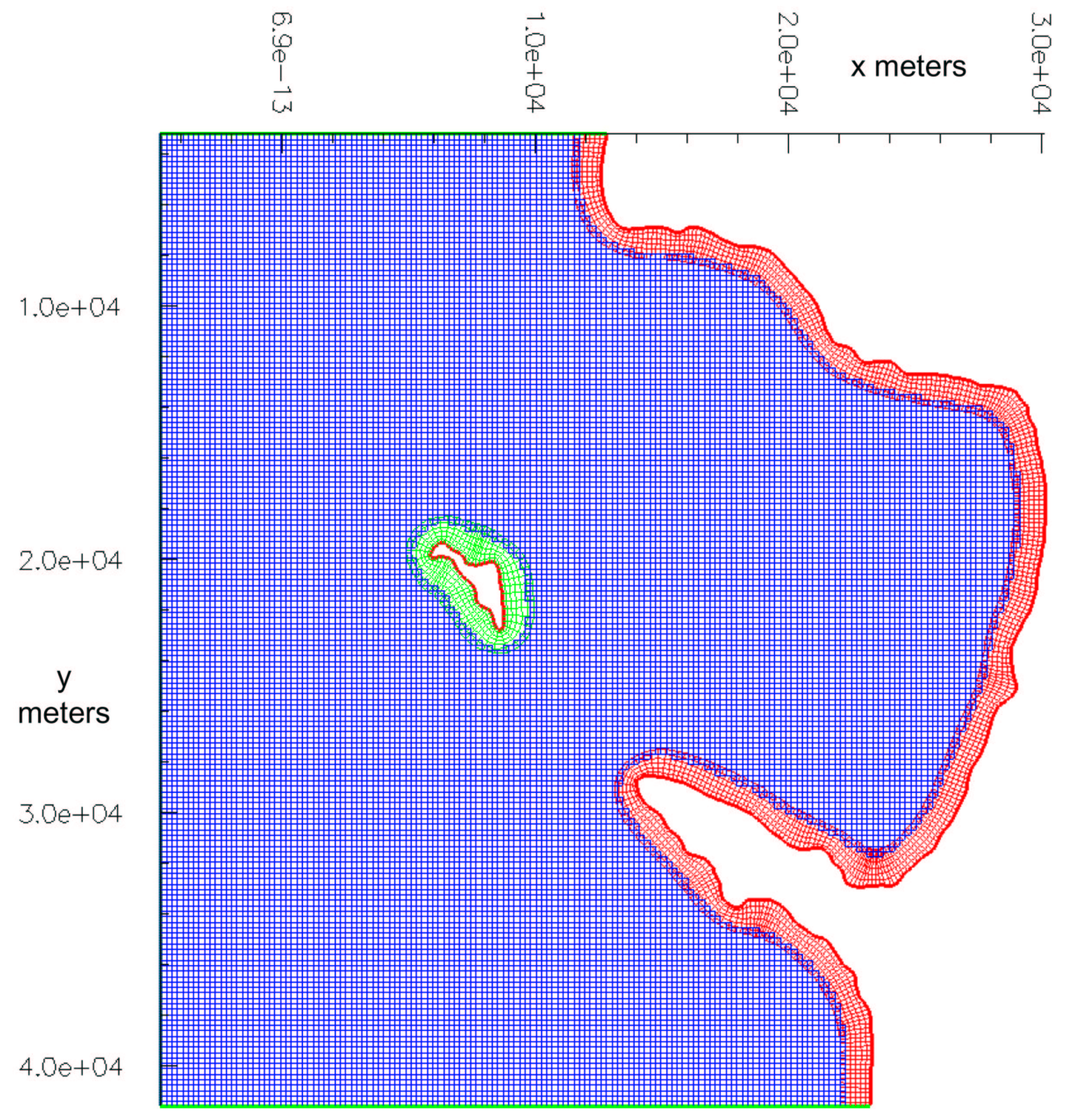

Figure 6.3: Overlapping grid of the Bahia de Todos Santos. 
Boundary conditions for the bay simulations are extrapolated from interior points on open boundaries except the western boundary for which the surface elevation is designated by the M2 tide (0.487 meter elevation, 12.42 hour period, and 270 degree phase). Along the coastline and island, nonslip boundary conditions are used. Wind forcing is used to drive the flow from the North in addition to the M2 tide, and the value is 0.034 $\mathrm{N} / \mathrm{m}^{2}$ as given by [9]. The Chézy friction coefficient used is $57.18 \mathrm{~m}^{1 / 2} / \mathrm{s}$, the depth is set to a constant 60 meters across the grids, and $\nu$ eddy viscosity is arbitrarily set at 10 $\mathrm{m}^{2} / \mathrm{s}$ to compare results from the previous study on the bay [9]. Results of the previous study on the bay are presented in Figure 6.4.

This simulation is run for 1,000,000 time steps of 0.2 seconds, which is roughly 55.6 hours. Simulations run on bays of this size are typically run for weeks or months of simulation time, so the run presented here is not considered to be completely past the transient phase. But the results shown here indicate a tendency towards a solution state that is dependent on the tides; this is the behavior expected for longer run simulations. The advective time constraint on the system due to relatively small grid spacing around the island and parts of the coastline make the simulation computationally expensive; the time taken to run the simulation results presented took seven days on a desktop Pentium Linux machine (unoptimized code).

One can see, comparing previous results with current results, that behavior is more detailed with the finer grid as grid spacing is reduced from 1 kilometer to 200 meters. However, the previous study includes the bottom bathymetry in the simulation whereas this study uses a constant depth. Therefore, a detailed comparison of results is not applicable. There are recirculation patters that emerge, seen in Figure 6.4, from the transition to low tide (as the water ebbs) apparent in this simulation that are not presented in previous study. These recirculation patters (the largest around the northern entrance) make sense in terms of physical behavior, but it is necessary to validate the computational scheme against the physics of the problem before any real scientific exploration can be gained here. 


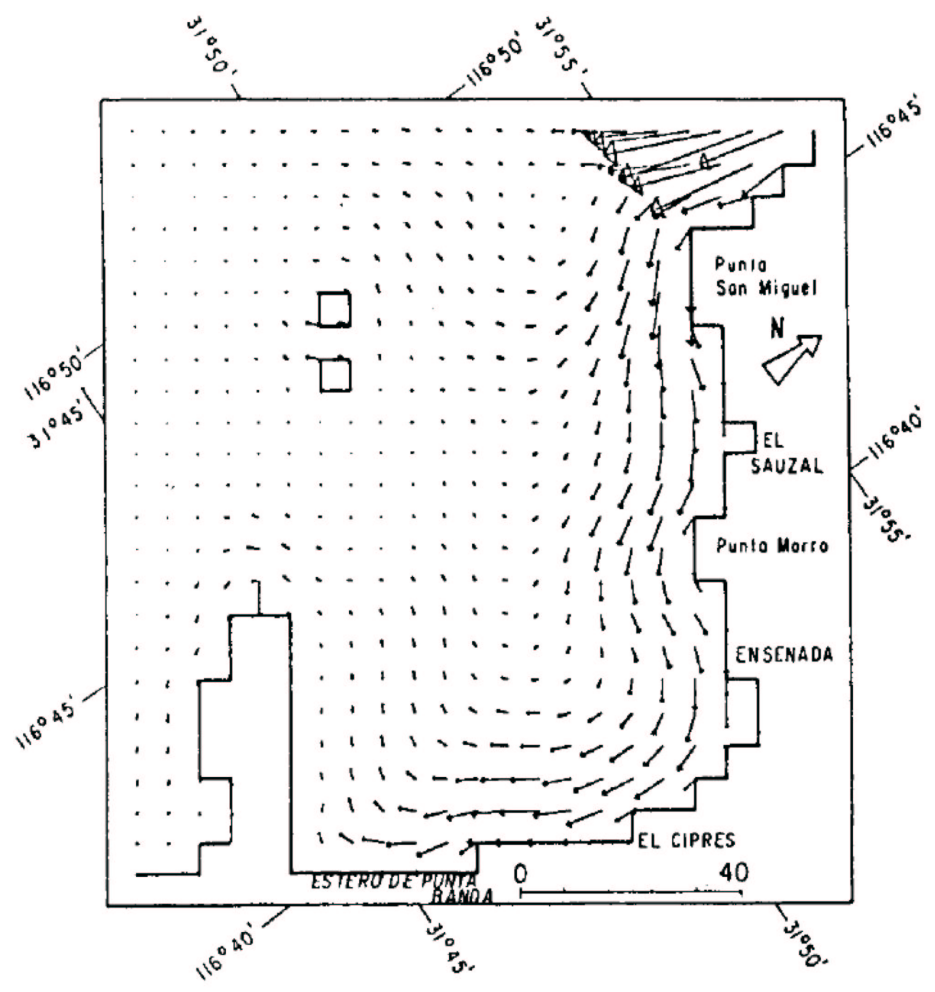

Figure 6.4: Results of previous simulation study of the Bahia de Todos Santos [9]. F.J. Gavidia Medina Espinoza, M.L.Argote and A. Amador Buenrostro. Wind- induced circulation in Todos Santos Bay, B.C., Mexico. Atmosphera, 4:101-115, 1991.

The arbitrary eddy viscosity coefficient chosen for this simulation directly affects the recirculation patters (as show by the flow past an idealized island example run), and no other means of selecting the value for the eddy viscosity coefficient is presented in this study. Another means of selecting this value, either by algebraic expression or by configuring it to reflect the actual behavior, needs to be included for this simulation to make any real, physical sense. It is evident, however, that there is potential for this application to have an impact on real scientific discovery for this bay, assuming bottom bathymetry is included. Figures 6.5 through 6.9 show results for other tidal stages over the 12.42 hour period: low tide, transition to high tide, high tide, and transition back to low tide again. 


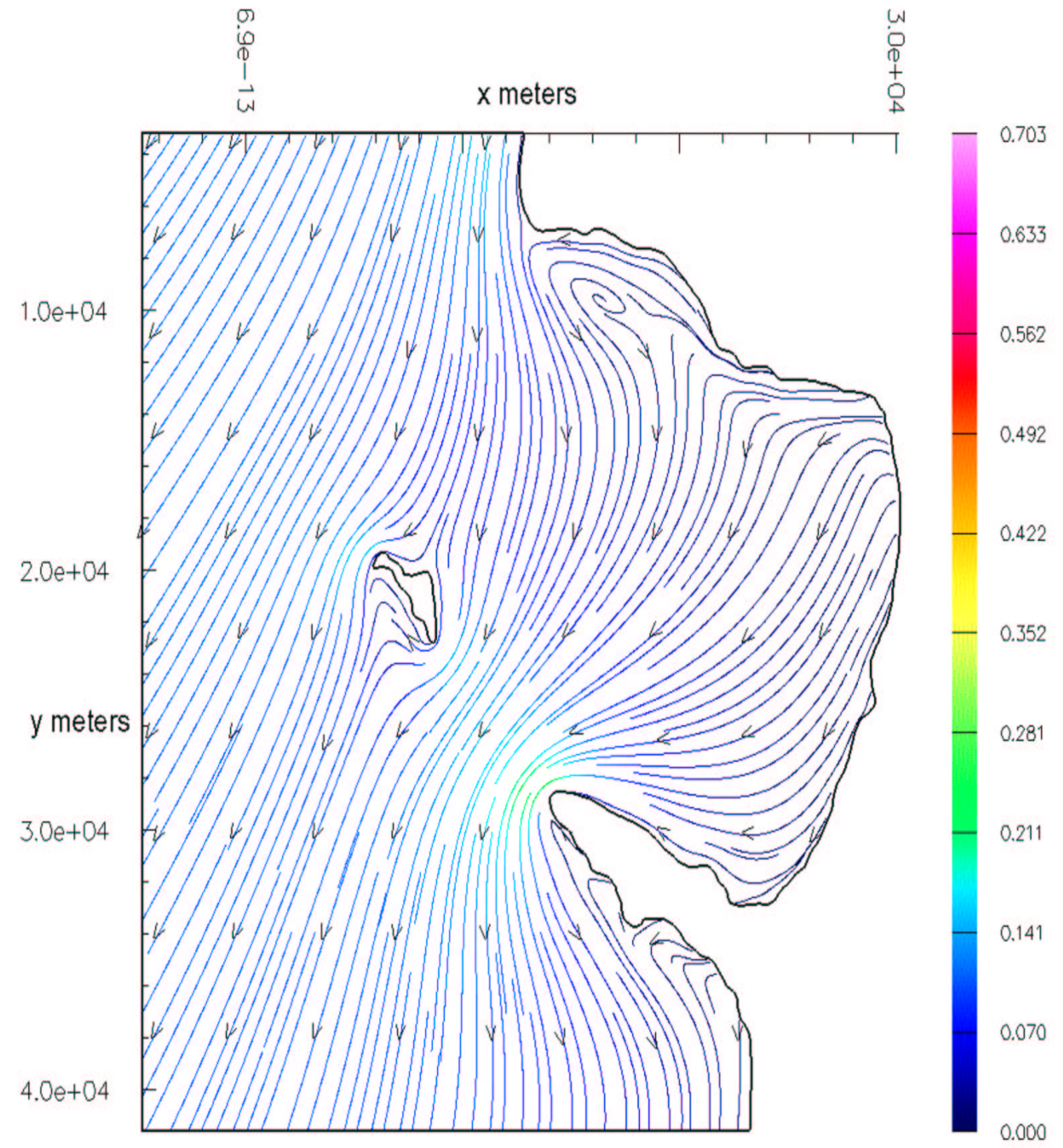

Figure 6.5: Flow through the bay forced by M2 tide and wind from the North. Flow is in a transition state moving from high tide to low tide, 40.6 hours. 


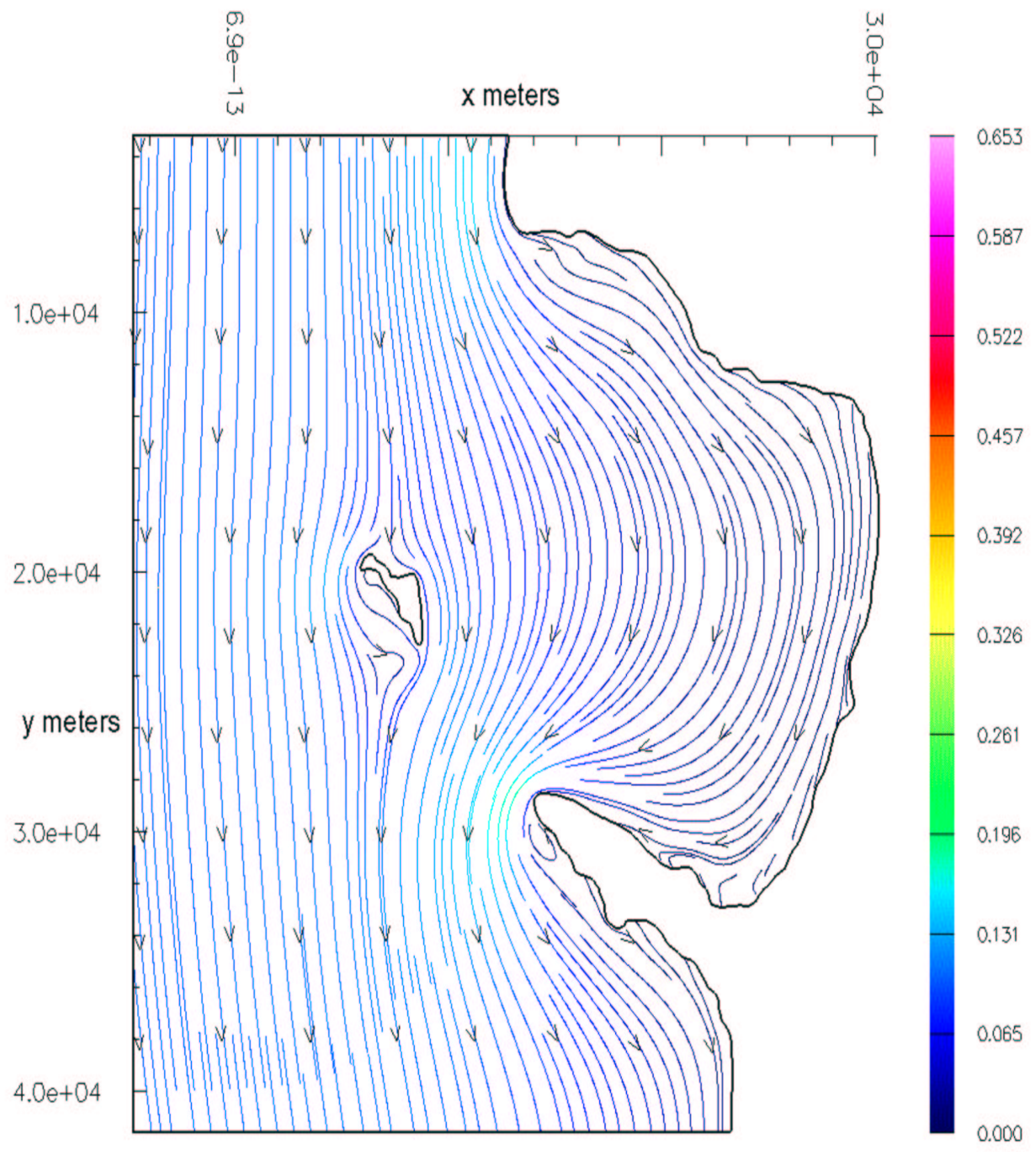

Figure 6.6: Flow through the bay. Flow is at low tide, 43.3 hours. 


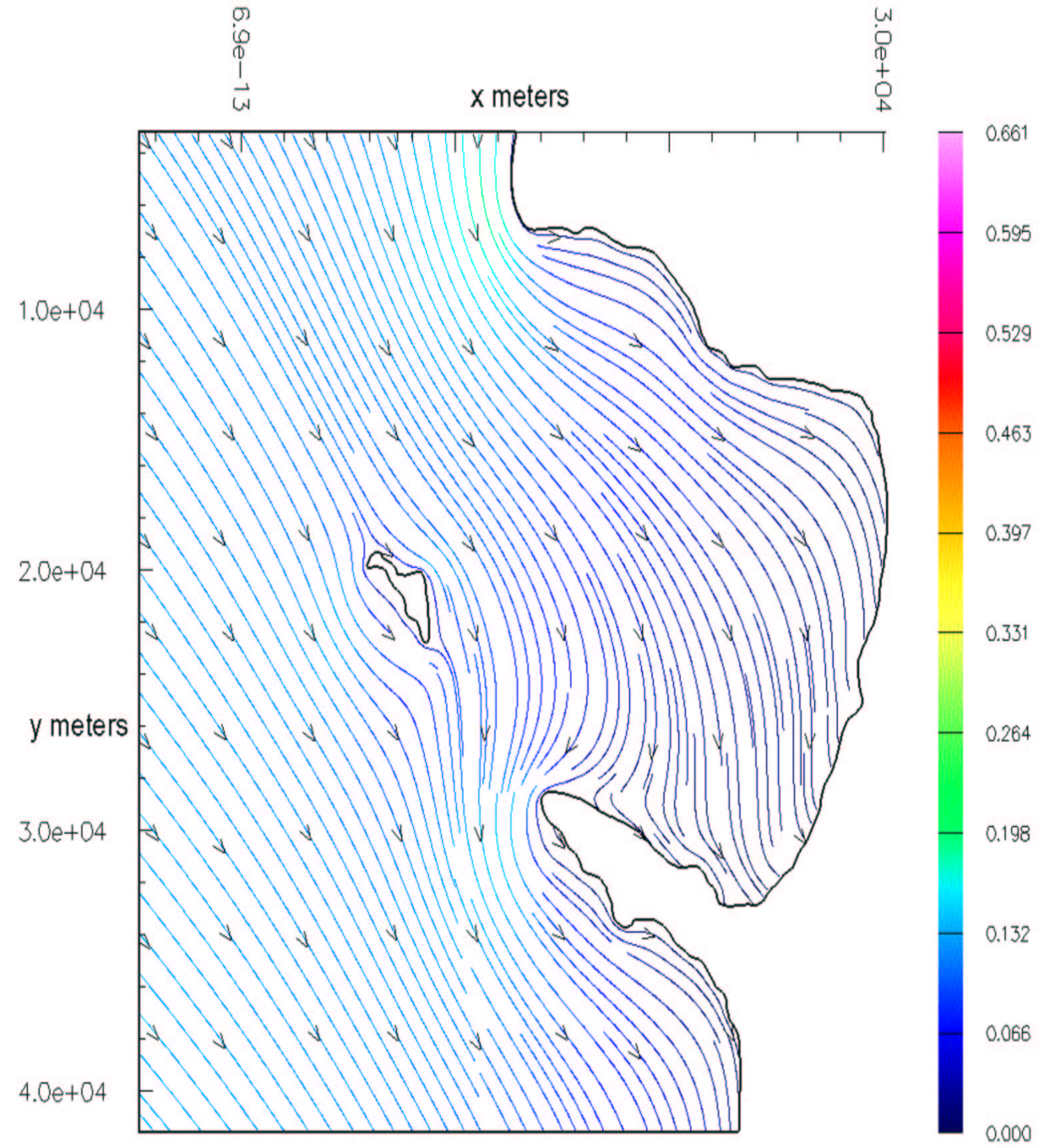

Figure 6.7: Flow through the bay. Flow is in a transition state moving from low tide to high tide, 46.7 hours. 


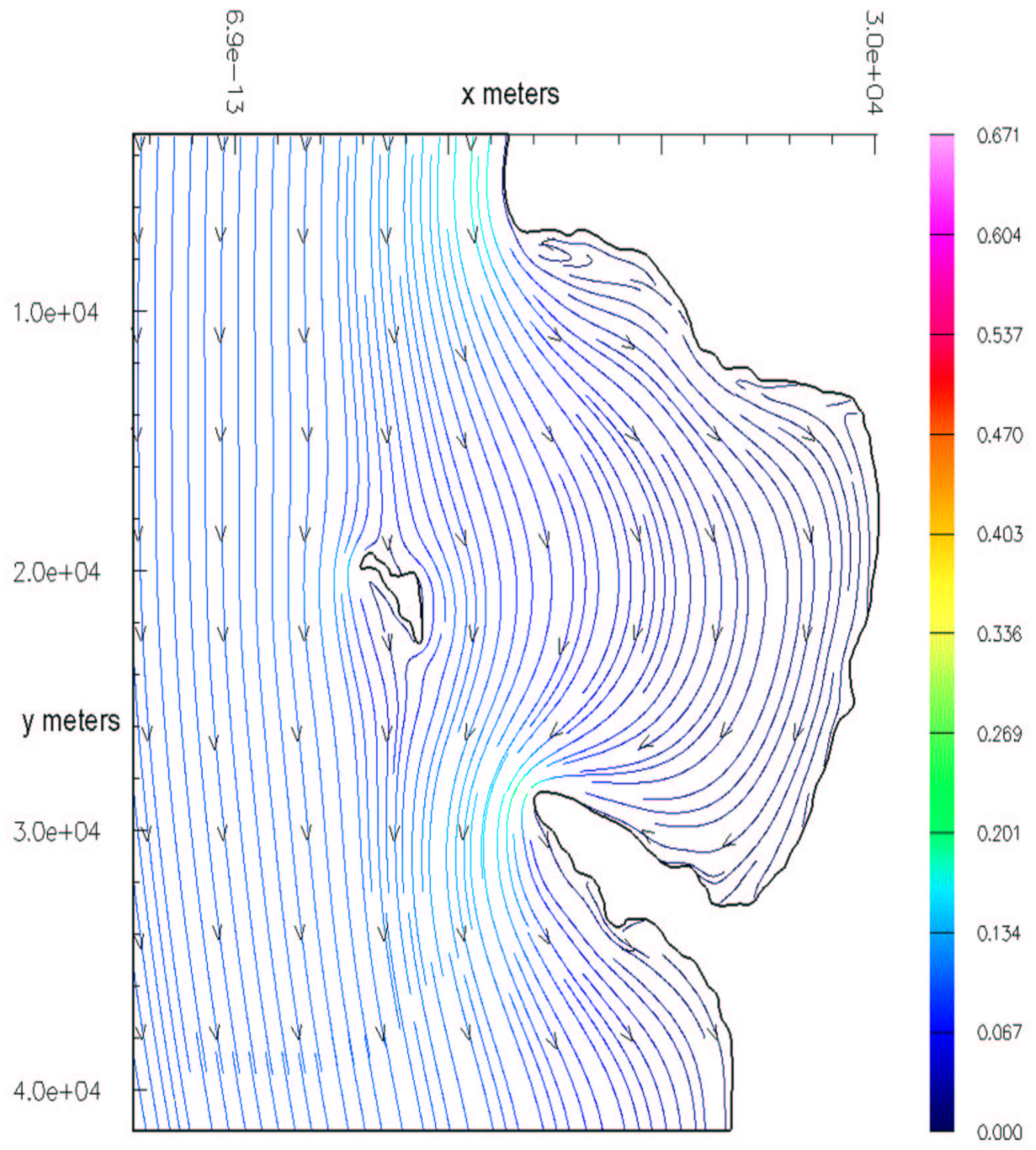

Figure 6.8: Flow through the bay. Flow is at high tide, 49.7 hours. 


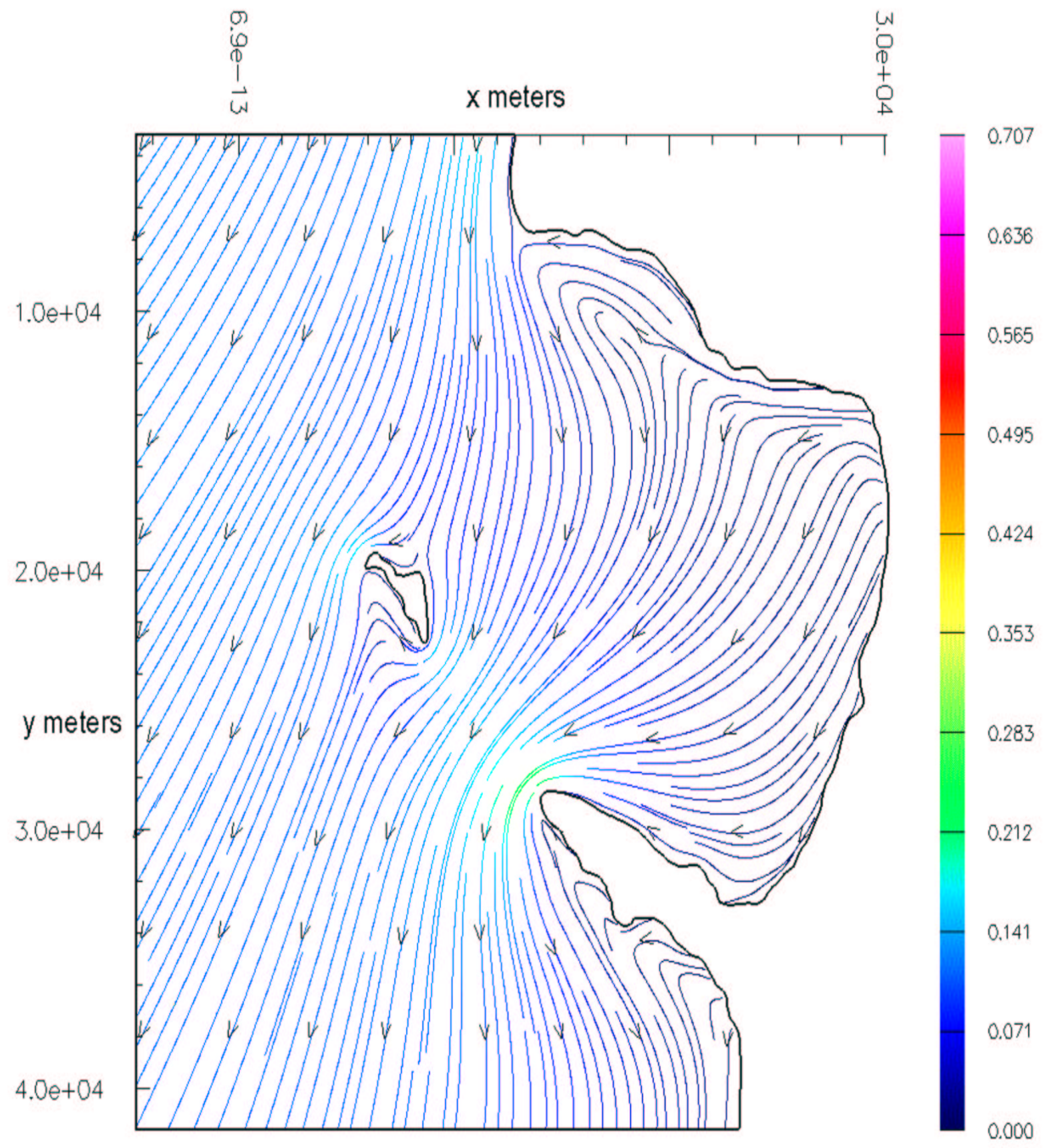

Figure 6.9: Flow through the bay. Flow is in transition from high tide to low tide, 52.8 hours. 


\section{CHAPTER 7}

\section{CONCLUSION}

There are many drawbacks when implementing any system of equations, both in the selection of the equations and in the discretization. The shallow water equations are limited to a certain set of physical problems in accordance with the assumptions mentioned in the introduction. There are also the limitations of computer space and speed, which even in this era of ever-increasing computational power, still limits problem size and complexity.

With this in mind, it is still possible to draw meaningful and interesting conclusions about the behavior of fluid flow on large scales by performing various simulations like the ones done here. While this study has mainly focused on the update of an original study done in the 1970s [23], and has neglected the important — and daunting — task of verification against field data. The reason for this is because this study is in computational science and not oceanography. This oversight should be forgiven by the attempts to choose some example and perform a mental check on the behavior, such as for the generic river, idealized island and flow over a seamount. The focus was to take the model and apply current techniques to expand the abilities of the solver; one that was chiefly accomplished when the equations were transformed into curvilinear coordinates.

This study initially started to implement the ADI technique to solve the transformed equations, but was eventually passed over in favor of a straight-forward explicit method. The problems with adapting the ADI method to suit the new transformed equations is that it is no longer clear which terms should be evaluated at the advanced time step and which at the current time step (and would require the prediction, probably by means of an iterative technique, of advanced time step values that could not be separated from the right hand side). Coupling this increase in complexity to the new inclusion of the viscosity terms of the momentum equations (which were not included in the original study), and the idea was dropped. The main drawback of the explicit method is the time step constraint which was thought an acceptable trade-off for the ease of creating 
an explicit finite difference scheme.

Despite the drawbacks mentioned here, however, there is potential for the method presented here to be used for valid scientific investigation. Any further study along these lines should begin with the addition of a more meaningful turbulence model. It seems impossible to appropriately describe the complexities of fluid flow without sufficiently managing the way viscosity affects the fluid with changes in velocity and depth. Likewise, boundary conditions and the effects of the flow near shore need further attention to attain a more realistic physical agreement. The use of artificial viscosity in the surface elevation equation for smoothing high frequency oscillation error (checker boarding) on complicated geometries should be avoided. Staggering the grid instead of using a fully center grid done here should aid this problem. 


\section{BIBLIOGRAPHY}

[1] C.M. Aiken and J.H. Middleton A.M. Moore. Non-normal perturbation growth in idealized island and headland wakes. Dynamics of Atmospheres and Oceans, 37:171$195,2003$.

[2] A. Balzano. Evaluation of methods for numerical simulation of wetting and drying in shallow water flow models. Coastal Engineering, 34:83-107, 1998.

[3] R.W. Barber. Numerical Modelling of Jet-Forced Circulation in Reservoirs Using Boundary-Fitted Coordinate Systems. PhD thesis, University of Salford, Salford, U.K., 1990.

[4] A.G.L Borthwick and R.W. Barber. River and reservoir flow modelling using the transformed shallow water equations. International Journal for Numerical Methods in Fluids, 14:1193-1217, 1992.

[5] D.L. Brown and D.J. Quinlan W.D. Henshaw, editors. Proceedings of the 17th AIAA Applied Aerodynamics Conference, number AIAA-99-3130 in AIAA, Norfolk, Virginia, 1999. American Institute of Aeronautics and Astronautics.

[6] R.T. Cheng and J.W. Gartner V. Casulli. Tidal, Residual, Intertidal Mudflat (TRIM) Model and its applications to San Francisco Bay, California. Estuarine, Coastal and Shelf Science, 36:235-280, 1993.

[7] G. Chesshire and W.D. Henshaw. Composite overlapping meshes for the solution of partial differential equations. Journal of Computational Physics, 90:1-64, 1990.

[8] Jacques Descloitres. Fires in Northern Baja California, Mexico (5 of 7). National Aeronautics and Space Administration (NASA) Visible Earth Website, 2003. Retrieved August 2004. http://visibleearth.nasa.gov/cgi-bin/viewrecord?25597.

[9] F.J. Gavidia Medina Espinoza, M.L.Argote and A. Amador Buenrostro. Windinduced circulation in Todos Santos Bay, B.C., Mexico. Atmosphera, 4:101-115, 1991.

[10] R.A. Falconer. Temperature distributions in tidal flow field. J. Hydraulics Division ASCE, 110:1099-1116, December 1984.

[11] C.A.J. Fletcher. Computational Techniques for Fluid Dynamics, volume 1 and 2. Springer, New York, 2 edition, 1991.

[12] K. Furukawa and E. Wolanski. Shallow-water frictional effects in island wakes. Estuarine, Coastal and Shelf Science, 46:599-608, 1998.

[13] K.A. Hoffmann and Steve T. Chiang. Computational Fluid Dynamics for Engineers, volume 3. Engineering Education System, Wichita, 4 edition, 2000. 
[14] K.A. Hoffmann and Steve T. Chiang. Computational Fluid Dynamics for Engineers, volume 1. Engineering Education System, Wichita, 4 edition, 2000.

[15] C.T. Hsu and J.C. Yang K.C. Yeh. Depth-averaged two-dimensional curvilinear explicit finite analytic model for open-channel flows. International Journal for $\mathrm{Nu}$ merical Methods in Fluids, 33:175-202, 2000.

[16] P. Knupp and S. Steinberg. Fundamentals of Grid Generation. CRC Press, Boca Raton, 1993.

[17] C.G. Koutitas. Mathematical Models in Coastal Engineering, chapter 2, pages 49-83. Pentech Press, London, 1988.

[18] G.H Lean and T.J. Weare. Modeling two-dimensional circulating flow. J. Hydraulics Division ASCE, 105:17-26, January 1979.

[19] J.J. Leendertse. Aspects of a computational model for long-period water wave propagation. Technical Report RM-5294-PR, The Rand Corporation, Santa Monica, CA, 1967.

[20] J.J. Leendertse. VAHM - a vertically averaged hydrodynamic model using boundary-fitted coordinates. Technical Report Misc. Paper HL-80-3, Army Engineer Waterways Experiment Station Hydraulics Laboratory, Vicksburg, MS, 1980.

[21] Randy LeVeque. Finite Volume Methods for Hyperbolic Problems. Cambridge University Press, New York, 2002.

[22] S.P. Neill and A.J. Elliot. In situ measurements of spring-neap variations to unsteady island wake development in the Firth of Forth, Scotland. Estuarine, Coastal and Shelf Science, 60:229-239, 2004.

[23] V.M. Ponce and S.B. Yabusaki. Modeling circulation in depth-averaged flow. J. Hydraulics Division ASCE, 11:1501-1518, 1981.

[24] P. Rao. A parallel hydrodynamic model for shallow water equations. Applied Mathematics and Computation, 150:291-302, 2004.

[25] L.J. Scott and R.W. Barber. The effects of grid adaption on tidal propagation in a boundary-fitted coordinate system. In N.P. Weatherill and J.F. Thompson P.R. Eiseman, J. Hauser, editors, Numerical Grid Generation in Computational Fluid Dynamics and Related Fields, pages 603-614. Pineridge Press Ltd., Mumbles, Swansea, UK, 1994.

[26] F. Shi and J.MK. Smith I.A Svendensen, J.T. Kirby. A curvilinear version of a quasi-3d nearshore circulation model. Coastal Engineering, 49:99-124, 2003.

[27] C.B Vreugdenhil and J.H.A. Wijbenga. Computation of flow patterns in rivers. J. Hydraulics Division ASCE, 108:1296-1310, November 1982. 
APPENDIX A

\section{TERM DEFINITIONS}




\section{TERM DEFINITIONS}

These are the term definitions, unless specifically stated otherwise by text:

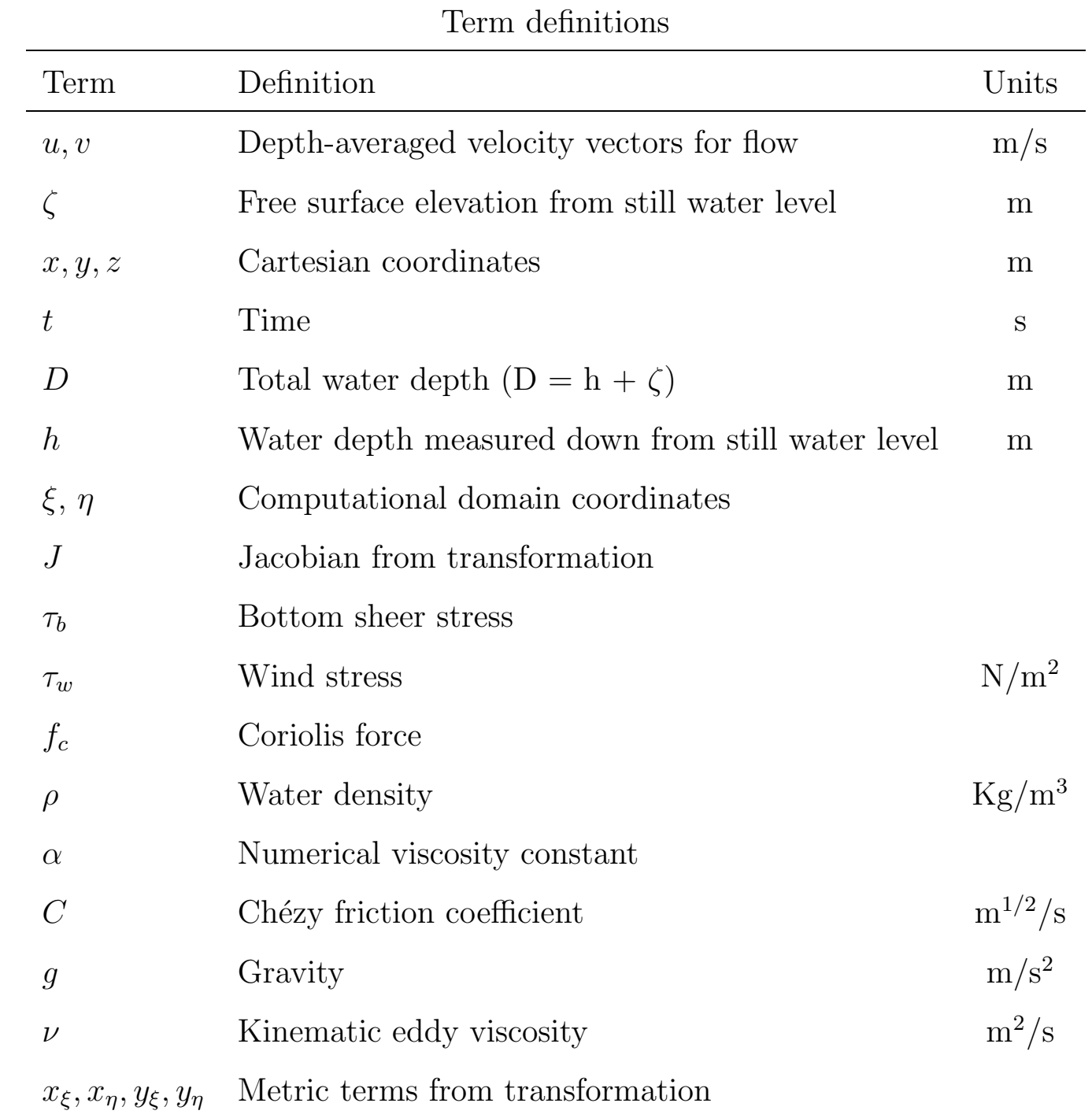


APPENDIX B

\section{FINITE DIFFERENCE SCHEME - $L$ OPERATORS}




\section{FINITE DIFFERENCE SCHEME - $L$ OPERATORS}

The simulation uses the method of lines. The Adams Predictor Corrector second

order finite difference scheme is used for the time step. All spatial derivatives are represented by second order, centered differences. For example,

$$
u_{\xi}^{n}=\frac{u_{j+1, k}^{n}+u_{j-1, k}^{n}}{2 \Delta \xi} .
$$

The Adams predictor step is,

$$
\begin{aligned}
& u_{j, k}^{p}=u_{j, k}{ }^{n}+\frac{\Delta t}{2}\left[3\left(L_{u}^{n}\right)_{j, k}-\left(L_{u}^{n-1}\right)_{j, k}\right], \\
& v_{j, k}^{p}=v_{j, k}^{n}+\frac{\Delta t}{2}\left[3\left(L_{v}^{n}\right)_{j, k}-\left(L_{v}^{n-1}\right)_{j, k}\right], \\
& \zeta_{j, k}^{p}=\zeta_{j, k}^{n}+\frac{\Delta t}{2}\left[3\left(L_{\zeta}^{n}\right)_{j, k}-\left(L_{\zeta}^{n-1}\right)_{j, k}\right] .
\end{aligned}
$$

And the Adams corrector step is,

$$
\begin{aligned}
& u_{j, k}^{n+1}=u_{j, k}^{n}+\frac{\Delta t}{2}\left[\left(L_{u}^{p}\right)_{j, k}+\left(L_{u}^{n}\right)_{j, k}\right], \\
& v_{j, k}^{n+1}=v_{j, k}^{n}+\frac{\Delta t}{2}\left[\left(L_{v}^{p}\right)_{j, k}+\left(L_{v}^{n}\right)_{j, k}\right], \\
& \zeta_{j, k}^{n+1}=\zeta_{j, k}^{n}+\frac{\Delta t}{2}\left[\left(L_{\zeta}^{p}\right)_{j, k}+\left(L_{\zeta}^{n}\right)_{j, k}\right],
\end{aligned}
$$

where, 


$$
\begin{aligned}
& L_{u}=\frac{1}{J}\left[y_{\eta} u u_{\xi}-y_{\xi} u u_{\eta}+x_{\xi} v u_{\eta}-y_{\eta} v u_{\xi}\right]+\frac{g}{J}\left[y_{\eta} \zeta_{\xi}-y_{\xi} \zeta_{\eta}\right]-f_{c} v-\frac{\tau_{w x}-\tau_{b x}}{\rho D}-T_{x}, \\
& L_{v}=\frac{1}{J}\left[y_{\eta} u v_{\xi}-y_{\xi} u v_{\eta}+x_{\xi} v v_{\eta}-y_{\eta} v v_{\xi}\right]+\frac{g}{J}\left[x_{\xi} \zeta_{\eta}-x_{\eta} \zeta_{\xi}\right]+f_{c} u-\frac{\tau_{w y}-\tau_{b y}}{\rho D}-T_{y}, \\
& L_{\zeta}=\frac{1}{J}\left[y_{\eta}\left(D u_{\xi}+u D_{\xi}\right)-y_{\xi}\left(D u_{\eta}+u D_{\eta}\right)+x_{\xi}\left(D v_{\eta}+v D_{\xi}\right)-x_{\eta}\left(D v_{\xi}+v D_{\eta}\right)\right],
\end{aligned}
$$

and where,

$$
\begin{aligned}
& T_{x}=\frac{1}{\rho D J}\left[y_{\eta}\left(T_{x x} D\right)_{\xi}-y_{\xi}\left(T_{x x} D\right)_{\eta}+x_{\xi}\left(T_{x y} D\right)_{\eta}-x_{\eta}\left(T_{x y} D\right)_{\xi}\right] \\
& T_{y}=\frac{1}{\rho D J}\left[y_{\eta}\left(T_{x y} D\right)_{\xi}-y_{\xi}\left(T_{x y} D\right)_{\eta}+x_{\xi}\left(T_{y y} D\right)_{\eta}-x_{\eta}\left(T_{y y} D\right)_{\xi}\right] .
\end{aligned}
$$




\section{ABSTRACT}




\begin{abstract}
This paper presents an exploration of a numerical scheme solving the curvilinear form of the depth averaged shallow water equations. The numerical method solves the depth averaged shallow water equations on curvilinear grids using an explicit, Adams predictor corrector, finite difference, time stepping scheme, with a special extension of the scheme to Overture. Overture is a software framework for writing composite grid solvers in $\mathrm{C}++$, developed by Lawrence Livermore National Laboratory. In order to verify the finite difference scheme is solving the equations correctly, resolution and time development studies are used to measure convergence rates. Several simplified physical test cases for single grids are presented to explore the behavior of the numerical method. An extension of the numerical method to Overture in order to implement composite grids is used to simulate Bahia de Todos Santos, Baja California, Mexico, and results for this simulation are presented.
\end{abstract}

